\title{
Heterosis and combining ability studies by Line $\times$ tester analysis for fruit biochemical, morpho-physiological, and yield traits governing shelf life in tomato (Solanum lycopersicum L.)
}

pavan MP ( $\sim$ pavanmpgubbi@gmail.com)

College of Agriculture Shimoga: University of Agricultural and Horticultural Sciences https://orcid.org/0000-0002-0302-6121

Gangaprasad S

College of Agriculture Shimoga: University of Agricultural and Horticultural Sciences

Dushyanthakumar B M

College of Agriculture Shimoga: University of Agricultural and Horticultural Sciences

Nagrajappa Adivappar

College of Agriculture Shimoga: University of Agricultural and Horticultural Sciences

Research Article

Keywords: Combining ability, Gene action, Heterosis, Line $\times$ Tester analysis, Shelf life, Tomato

Posted Date: December 28th, 2021

DOI: https://doi.org/10.21203/rs.3.rs-1165702/v1

License: (c) (1) This work is licensed under a Creative Commons Attribution 4.0 International License. Read Full License 


\section{Abstract}

Improving tomatoes keeping quality is crucial for reducing post-harvest losses. Knowledge on heterosis, and combining ability is pre requisite for breeding high yielding and good shelf life heterotic hybrids. An investigation was undertaken with each of 3 lines, testers, and 9 hybrids to identify desirable parents and crosses for 20 fruit biochemical, morpho-physiological, and yield traits and to elucidate nature of gene action for shelf life and its contributing traits through Line $\times$ Tester analysis. The lines contributed to most of hybrids variability than testers and fruit quality traits had higher degree of SCA variance as compared to GCA variance. pH, ascorbic acid, fruit firmness, and plant height governed by additive gene action. Lycopene, titratable acidity, TSS, calcium, magnesium, pericarp thickness, pulp content, locule number, fruit length, diameter, weight, shelf life, number of branches, number of clusters, number of fruit/cluster, and yield/plant were under the control of non-additive gene action. All the lines and Arka Saurabh were best general combiners and IIHR $2349 \times$ Arka Vikas, IIHR $2349 \times$ Arka Saurabh, IIHR $2358 \times$ Arka Ahuti and IIHR $2357 \times$ Arka Ahuti were the best specific combiner in producing heterotic hybrids. IIHR $2349 \times$ Arka Vikas and IIHR $2349 \times$ Arka Saurabh were promising hybrids for high yield and shelf life. The crosses involved both parents with high, one parent with high and other with low and both parents with low good overall general combining ability status respectively indicated the additive, non-additive and epistatic gene action in fruit quality and yield traits inheritance.

\section{Introduction}

Tomato [(Solanum lycopersicum L.), $(2 \mathrm{n}=2 \mathrm{x}=24)$ ], is an important solanaceous vegetable crop in the world in terms of cultivation and consumption. It is nutritionally enriched with blood purification and anti-cancerous properties of antioxidants like lycopene, $\beta$-carotene, ascorbic acid, folic acid, phenolic acids, and flavonoids (Arab and Steck 2000; Freeman and Reimers 2011). It is a perishable vegetable with a relatively short shelf life, premature ripening and softening resulted in significant post-harvest losses. The post-harvest losses of tomatoes were estimated up to $25-42 \%$ in the world (Arah et al. 2015) and up to $50 \%$ in developing countries (Delina and Mahendran 2009).

The short shelf life of fruits is the result of improper storage, inadequate transportation, inefficient processing and preservation facilities. This leads to the occurrence of many chemical and physical changes in fruit viz., loss of weight, sugar and acid contents, respiration, softening of pulp and microbial decay greatly contribute to high post-harvest losses (Garcia et al. 2019). Consequently, huge volumes of tomatoes are sold at devalued prices and farmers get poor returns (Sinha 2019). Later, this period is succeeded by the fruit scarcity period with a high price in the market and consumers will suffer (Delina and Mahendran 2009).

The post-harvest shelf life is a prerequisite for commercially grown tomatoes. Extending the shelf life of tomatoes is highly essential for profitable marketing and reduction of great losses in quality and quantity (Salliba et al. 2001), as it gives more time to farmers for marketing before fruit quality is degraded (Osei et al. 2020). Therefore, reduction of post-harvest losses is the 'Need of the hour'. Even though many post-harvest preservation methods, chemical treatments, and anti-sense RNA technology are efficient in extending the shelf life but these technologies are not sufficient, practically infeasible in farmer's fields and they need social acceptance too. Therefore, genetic improvement of fruit quality traits seems to be the safest way to improve shelf life (Yogendra and Gowda 2013) and it is the 'Solution of the hour'.

Fruit shelf life is a ripening-related complex trait affected by several low inheritable fruit quality and yield traits. The desire for progress in crop improvement through plant breeding is propelled by a better understanding and appropriate exploitation of heterosis which is the gain in vigor on crossing two lines or varieties. Developing tomato hybrids for both yield as well as high shelf life is extremely essential to keep the tomato production and supply sustainable for the growing population in the present and near future. The hybrid performance depends on the choice of parents and parents can be selected based on per se performance, genetic diversity, combining ability, etc. Selection of parents based on per se performance alone is not a sound procedure since superior lines identified on this basis may yield poor recombinants in the segregating generations (Garg et al. 2007). The identification of the best combiners is a prerequisite for heterosis breeding. Therefore, success in hybrid development with desired traits hinges on the knowledge of combining ability, nature, and magnitude of gene action in different genetic backgrounds (Somraj et al. 2018).

The combining ability analysis offers a powerful tool to assess the genetic potential of parents in hybrid combination through general and specific combining abilities to produce a superior heterotic hybrid. Among many available statistical tools to detect the combining ability and gene action governing various quantitative traits, Line $\times$ Tester analysis has been a very useful design. With this justified focus, the present study was planned and executed with twin objectives of identification of desirable parents (combiners) and crosses (combinations) for fruit quality traits and to elucidate the genetics of less pursued but very important shelf life and its contributing traits through Line $\times$ Tester analysis in tomato.

\section{Materials And Methods}

The experiment consists of two steps, i.e. 1. Development of $F_{1}$ generations (Hybrids) and 2. Evaluation of lines, testers, and $F_{1}$ generations.

\subsection{Experimental site}

The present investigation was carried out by conducting the field and lab experiments during the 2017 summer and rainy seasons at the Department of Genetics and Plant Breeding, Keladi Shivappa Nayaka University of Agricultural and Horticultural Sciences, Navule, Shivamogga (13.9739 $\left.{ }^{0} \mathrm{~N}, 75.5791{ }^{0} \mathrm{E}\right)$, Karnataka, India.

\subsection{Basic genetic material}


The basic material for the study consisted of parents contrasting fruit shelf life and yield traits. The high shelf life low yielding genotypes viz., IIHR 2349 , IIHR 2357, IIHR 2358, and high yielding low shelf life varieties viz., Arka Vikas, Arka Ahuti, and Arka Saurabh were used as lines and testers respectively. For comparative analysis, 'Arka Rakshak', a popular public bred high yielding tomato hybrid was used as a standard check. The Lines, testers and check hybrid were maintained and released from ICAR-Indian Institute of Horticultural Research, Bengaluru, Karnataka, India.

\subsection{Development of experimental material}

The crosses were affected between high shelf life low yielding lines and high yielding low shelf life testers in Line $\times$ Tester $(3 \times 3)$ mating design. The nine first filial generations were developed by pollinating the pollens from testers onto the stigmas of emasculated flowers of lines in the early morning hours (6-8 AM) in protected poly house conditions during the 2017 summer. The seeds of lines, testers, and $F_{1}$ 's were harvested individually which constituted the experimental material.

\subsection{Evaluation of experimental material}

The nine hybrids along with their parents and standard check were planted in the field during the 2017 rainy season in two separate contiguous blocks in Randomized Complete Block Design with two replications. The standard crop production and protection practices were followed to raise healthy plants and were evaluated for fruit biochemical, morpho-physiological, and yield traits governing shelf life.

\subsection{Sampling of plants and collection of data}

The data were recorded from five randomly selected plants avoiding border plants in each line, testers, and $\mathrm{F}_{1}$ generations. The 20 fruit quality traits governing shelf life such as 7 fruit biochemical traits viz., TSS (\%) (using Erma hand refractometer), pH (using Siemens pH meter), lycopene (mg/100g) (Lichtenthaler 1987), ascorbic acid (mg/100g) (2,6-Dichlorophenol indophenol method, Association of Official Analytical Chemists 2006), titratable acidity (\%) (Association of Official Analytical Chemists 2000), calcium and magnesium content (mg/100g) (di-acid digestion and ICP-OES method), 8 morphophysiological traits viz., fruit length $(\mathrm{cm})$, fruit diameter $(\mathrm{cm})$ and pericarp thickness $(\mathrm{mm})$ were measured with digital vernier caliper, fruit weight $(\mathrm{g})$ (using digital weighing balance), fruit firmness $\left(\mathrm{kg} / \mathrm{cm}^{2}\right.$ ) (using fruit penetrometer), pulp content (\%) (Percent ratio of weight of pulp after removing fruit juice and seed to the fruit weight), locule number (manually counted), shelf life (Days) (counted as number of days taken by fruits harvested at breaker stage kept on shelf to show first visible shrinkage on its fruit surface) and 5 yield attributing traits viz., plant height (cm) (using measuring scale), number of branches, number of clusters, number of fruit/cluster were manually counted, yield/plant (g) (using digital weighing balance) were recorded in the field during harvest. The fruit quality traits were recorded in the lab at the red ripe stage of five randomly selected tomato fruits from each plant and the mean was computed.

\subsection{Statistical analysis}

The collected mean data on fruit quality traits of parents and hybrids were analyzed for percent heterosis over their mid-parent (mid parent heterosis or average heterosis), better parent (heterobeltiosis) and standard check (standard heterosis) (Turner 1953; Hayes et al. 1955), Analysis of Variance (ANOVA) (Panse and Sukhatme 1967), General Combining Ability (GCA) effects of lines, testers and Specific Combining Ability (SCA) effects of hybrids using Line $\times$ Tester analysis (Kempthorne 1957; Arunachalam 1974), percent contribution of lines, testers and their interaction towards total variability in each character (Singh and Choudhory 1977), the overall status of a parent or a cross concerning GCA or SCA effects (Arunachalam and Bandopadhyay 1979) with slight modification as suggested by Mohan Rao (2001) using 'WINDOSTAT' statistical package.

\section{Results}

\subsection{Mean performance of lines, testers, and hybrids for fruit biochemical, morpho- physiological, and yield attributing traits}

The lines and testers recorded substantial variability for all traits. The performance of lines was higher compared to testers for $\mathrm{pH}, \mathrm{calcium}$, magnesium, fruit firmness, pericarp thickness, pulp content, locule number, shelf life, and number of clusters (Table I). Line IIHR 2349 was superior for pH, titratable acidity, calcium, magnesium, fruit length, diameter, weight, pulp content, number of branches, and number of clusters. Similarly, IIHR 2357 recorded higher TSS, lycopene, magnesium, number of fruits and yield/plant and IIHR 2358 exhibited higher ascorbic acid, fruit firmness, pericarp thickness, shelf life, and plant height. Among the testers, Arka Vikas recorded better mean for TSS, ascorbic acid, titratable acidity, magnesium, fruit diameter, locule number, number of clusters, number of fruits, and yield/plant. Arka Ahuti recorded higher $\mathrm{pH}$, fruit length, firmness, pericarp thickness, pulp content, shelf life, and number of branches. Arka Saurabh exhibited higher lycopene, calcium, fruit weight, and plant height.

The hybrids were superior in their performance than parents for lycopene, fruit diameter, weight, firmness, pericarp thickness, locule number, plant height, number of clusters, number of fruits, and yield/plant.

Table I: Estimates of mean performance of tomato lines, testers and hybrids for fruit biochemical, morpho-physiological and yield attributing traits 


\begin{tabular}{|c|c|c|c|c|c|c|c|c|c|c|c|}
\hline $\begin{array}{l}\text { SI. } \\
\text { No. }\end{array}$ & Genotypes & $\begin{array}{l}\text { TSS } \\
(\%)\end{array}$ & $\mathrm{pH}$ & $\begin{array}{l}\text { Lycopene } \\
\text { (mg/100g) }\end{array}$ & $\begin{array}{l}\text { Ascorbic acid } \\
(\mathrm{mg} / 100 \mathrm{~g})\end{array}$ & $\begin{array}{l}\text { Titratable } \\
\text { acidity (\%) }\end{array}$ & $\begin{array}{l}\text { Calcium } \\
(\mathrm{mg} / 100 \mathrm{~g})\end{array}$ & $\begin{array}{l}\text { Magnesium } \\
(\mathrm{mg} / 100 \mathrm{~g})\end{array}$ & $\begin{array}{l}\text { Fruit } \\
\text { length } \\
\text { (cm) }\end{array}$ & $\begin{array}{l}\text { Fruit } \\
\text { diameter } \\
\text { (cm) }\end{array}$ & $\begin{array}{l}\text { Fruit } \\
\text { weight } \\
\text { (g) }\end{array}$ \\
\hline \multicolumn{12}{|c|}{ Lines } \\
\hline 1 & IIHR 2349 & 2.50 & 5.52 & 2.49 & 7.15 & 0.77 & 16.16 & 13.24 & 45.76 & 40.73 & 42.00 \\
\hline 2 & IIHR 2358 & 2.80 & 5.48 & 0.52 & 17.73 & 0.42 & 15.81 & 10.73 & 35.17 & 34.61 & 23.40 \\
\hline 3 & IIHR 2357 & 3.20 & 5.50 & 2.61 & 8.78 & 0.47 & 16.16 & 13.24 & 42.39 & 37.15 & 33.00 \\
\hline \multicolumn{12}{|c|}{ Testers } \\
\hline 4 & Arka Vikas & 3.20 & 5.27 & 0.80 & 23.90 & 0.83 & 11.15 & 14.43 & 38.37 & 42.15 & 45.50 \\
\hline 5 & Arka Ahuti & 3.15 & 5.55 & 2.44 & 7.11 & 0.67 & 13.10 & 8.08 & 56.95 & 38.57 & 44.80 \\
\hline 6 & Arka Saurabh & 3.00 & 5.5 & 3.65 & 7.31 & 0.73 & 13.81 & 11.13 & 45.76 & 38.72 & 47.16 \\
\hline \multicolumn{12}{|c|}{ Hybrids } \\
\hline 7 & $\begin{array}{l}\text { IIHR } 2349 \text { x } \\
\text { Arka Vikas }\end{array}$ & 2.50 & 5.43 & 2.04 & 10.76 & 0.67 & 11.29 & 5.29 & 44.12 & 45.27 & 55.70 \\
\hline 8 & $\begin{array}{l}\text { IIHR } 2349 x \\
\text { Arka Ahuti }\end{array}$ & 2.50 & 5.41 & 1.94 & $17.65^{\star \star}$ & 0.59 & 5.71 & 5.12 & 39.67 & 45.38 & 47.10 \\
\hline 9 & $\begin{array}{l}\text { IIHR } 2349 x \\
\text { Arka Saurabh }\end{array}$ & 2.95 & 5.51 & $4.59 * \star$ & 7.43 & 0.67 & 17.99 & $17.79 * *$ & 51.40 & 45.14 & 58.40 \\
\hline 10 & $\begin{array}{l}\text { IIHR } 2358 \text { x } \\
\text { Arka Vikas }\end{array}$ & 2.15 & 5.51 & 2.09 & 7.18 & 0.67 & 13.79 & $10.93^{\star \star}$ & 42.39 & 40.55 & 37.80 \\
\hline 11 & $\begin{array}{l}\text { IIHR } 2358 \text { x } \\
\text { Arka Ahuti }\end{array}$ & 2.30 & 5.42 & 1.73 & 14.38 & 0.54 & 14.63 & $13.03^{\star \star}$ & 49.14 & 48.22 & 64.50 \\
\hline 12 & $\begin{array}{l}\text { IIHR } 2358 \text { x } \\
\text { Arka Saurabh }\end{array}$ & 2.90 & 5.38 & 0.91 & 7.38 & 0.67 & 11.72 & $13.79 * *$ & 51.73 & 43.41 & 50.40 \\
\hline 13 & $\begin{array}{l}\text { IIHR } 2357 \text { x } \\
\text { Arka Vikas }\end{array}$ & 2.40 & 5.37 & 4.22 ** & 7.38 & 0.20 & 9.48 & 6.55 & 41.95 & 46.09 & 50.60 \\
\hline 14 & $\begin{array}{l}\text { IIHR } 2357 \text { x } \\
\text { Arka Ahuti }\end{array}$ & 2.45 & 5.15 & 1.25 & 13.75 & 0.54 & 14.20 & $12.75^{\star \star}$ & 47.16 & 45.95 & 61.75 \\
\hline 15 & $\begin{array}{l}\text { IIHR } 2357 \text { x } \\
\text { Arka Saurabh }\end{array}$ & 2.25 & 5.44 & $3.23^{\star *}$ & 11.11 & 0.25 & 14.39 & $14.24^{\star \star}$ & 40.11 & 43.08 & 41.20 \\
\hline 16 & $\begin{array}{l}\text { Arka Rakshak } \\
\text { (check) }\end{array}$ & 3.00 & 5.70 & 2.01 & 13.70 & 0.54 & 19.35 & 6.99 & 55.58 & 45.79 & 63.20 \\
\hline \multicolumn{2}{|c|}{ Mean } & 2.49 & 5.40 & 2.44 & 10.78 & 0.53 & 12.58 & 11.05 & 45.30 & 44.79 & 51.94 \\
\hline \multicolumn{2}{|c|}{ S.E. \pm} & 0.21 & 0.07 & 0.10 & 0.34 & 0.08 & 0.01 & 0.05 & 2.07 & 1.93 & 3.51 \\
\hline \multicolumn{2}{|c|}{ C.D. $5 \%$} & 0.48 & 0.15 & 0.23 & 0.79 & 0.19 & 0.01 & 0.13 & 4.77 & 4.46 & 8.10 \\
\hline \multicolumn{2}{|c|}{ C.D. $1 \%$} & 0.70 & 0.22 & 0.33 & 1.14 & 0.28 & 0.01 & 0.18 & 6.95 & 6.49 & 11.78 \\
\hline
\end{tabular}

Cont'd... (Table l: Estimates of mean performance of tomato lines, testers and hybrids for fruit biochemical, morpho-physiological and yield attributing traits) 


\begin{tabular}{|c|c|c|c|c|c|c|c|c|c|c|c|}
\hline $\begin{array}{l}\text { SI. } \\
\text { No. }\end{array}$ & Genotypes & $\begin{array}{l}\text { Fruit } \\
\text { firmness } \\
\left(\mathrm{kg} / \mathrm{cm}^{2}\right)\end{array}$ & $\begin{array}{l}\text { Pericarp } \\
\text { thickness } \\
(\mathrm{mm})\end{array}$ & $\begin{array}{l}\text { Pulp } \\
\text { content } \\
\text { (\%) }\end{array}$ & $\begin{array}{l}\text { Locule } \\
\text { number }\end{array}$ & $\begin{array}{l}\text { Shelf } \\
\text { life } \\
\text { (Days) }\end{array}$ & $\begin{array}{l}\text { Plant } \\
\text { height } \\
\text { (cm) }\end{array}$ & $\begin{array}{l}\text { No. of } \\
\text { branches }\end{array}$ & $\begin{array}{l}\text { No. of } \\
\text { clusters }\end{array}$ & $\begin{array}{l}\text { No. of } \\
\text { fruit/ } \\
\text { cluster }\end{array}$ & $\begin{array}{l}\text { Yield/ } \\
\text { plant (g) }\end{array}$ \\
\hline \multicolumn{12}{|c|}{ Lines } \\
\hline 1 & IIHR 2349 & 2.01 & 4.35 & 82.14 & 3.00 & 25.10 & 57.20 & 4.60 & 11.15 & 3.40 & 606.15 \\
\hline 2 & IIHR 2358 & 2.47 & 4.90 & 76.53 & 3.00 & 28.85 & 57.30 & 4.30 & 10.70 & 3.50 & 667.80 \\
\hline 3 & IIHR 2357 & 2.13 & 4.48 & 81.39 & 3.00 & 27.40 & 56.70 & 4.20 & 7.70 & 4.00 & 831.90 \\
\hline \multicolumn{12}{|c|}{ Testers } \\
\hline 4 & Arka Vikas & 1.68 & 4.24 & 71.10 & 3.50 & 17.10 & 64.20 & 4.30 & 11.80 & 4.40 & 1155.50 \\
\hline 5 & Arka Ahuti & 1.91 & 4.76 & 83.29 & 2.00 & 20.05 & 64.70 & 5.20 & 7.10 & 3.40 & 1037.00 \\
\hline 6 & Arka Saurabh & 1.90 & 3.82 & 80.52 & 3.00 & 17.55 & 71.70 & 4.20 & 7.70 & 3.50 & 1006.06 \\
\hline \multicolumn{12}{|c|}{ Hybrids } \\
\hline 7 & $\begin{array}{l}\text { IIHR } 2349 \text { x } \\
\text { Arka Vikas }\end{array}$ & 2.60 & 5.63 & 76.64 & 3.35 & 24.70 & 66.80 & 3.80 & $11.05^{\star \star}$ & 4.15 & 2850.50 * \\
\hline 8 & $\begin{array}{l}\text { IIHR } 2349 \text { x } \\
\text { Arka Ahuti }\end{array}$ & 2.05 & 4.60 & 70.82 & 3.30 & 20.10 & 74.00 & 4.80 & 9.70 & 5.15 & 2190.55 \\
\hline 9 & $\begin{array}{l}\text { IIHR } 2349 \text { x } \\
\text { Arka Saurabh }\end{array}$ & 2.08 & 5.09 & 78.38 & 3.50 & $29.40 *$ & 64.10 & 4.10 & 10.15 & 4.35 & 2397.50 \\
\hline 10 & $\begin{array}{l}\text { IIHR } 2358 \text { x } \\
\text { Arka Vikas }\end{array}$ & 2.55 & 4.71 & 84.68 & 3.45 & 21.50 & 58.80 & 4.60 & 8.80 & 4.35 & 2064.50 \\
\hline 11 & $\begin{array}{l}\text { IIHR } 2358 \text { x } \\
\text { Arka Ahuti }\end{array}$ & 2.32 & 5.05 & 75.99 & 3.35 & 22.60 & 83.00 ** & 3.60 & $11.25^{\star \star}$ & 5.15 & 2109.75 \\
\hline 12 & $\begin{array}{l}\text { IIHR } 2358 \text { x } \\
\text { Arka Saurabh }\end{array}$ & 2.26 & 3.50 & 82.63 & 3.65 & 26.60 & 67.90 & 4.50 & 9.70 & 3.15 & 2005.50 \\
\hline 13 & $\begin{array}{l}\text { IIHR } 2357 \text { x } \\
\text { Arka Vikas }\end{array}$ & 2.69 & 4.76 & 82.22 & 3.35 & $28.00 *$ & 57.60 & 4.20 & 7.05 & 3.60 & 2103.25 \\
\hline 14 & $\begin{array}{l}\text { IIHR } 2357 \text { x } \\
\text { Arka Ahuti }\end{array}$ & 2.25 & 4.55 & 75.90 & 3.35 & 22.80 & $76.75^{\star \star}$ & 2.45 & $10.95^{\star \star}$ & 5.15 & 2057.35 \\
\hline 15 & $\begin{array}{l}\text { IIHR } 2357 x \\
\text { Arka Saurabh }\end{array}$ & 1.77 & 4.59 & 80.19 & 3.00 & 24.20 & 72.60 & 5.10 & $10.85^{\star \star}$ & $5.65^{\star}$ & 2117.75 \\
\hline 16 & $\begin{array}{l}\text { Arka Rakshak } \\
\text { (check) }\end{array}$ & 3.01 & 5.93 & 83.46 & 2.50 & 23.45 & 74.70 & 4.00 & 10.15 & 5.35 & 2389.65 \\
\hline \multicolumn{2}{|c|}{ Mean } & 2.29 & 4.72 & 78.61 & 3.37 & 24.43 & 69.09 & 4.13 & 9.94 & 4.52 & 2130.77 \\
\hline \multicolumn{2}{|c|}{ S.E. \pm} & 0.28 & 0.56 & 4.94 & 0.51 & 2.21 & 0.07 & 0.76 & 0.15 & 0.10 & 193.56 \\
\hline \multicolumn{2}{|c|}{ C.D. $5 \%$} & 0.64 & 1.29 & 11.40 & 1.19 & 4.31 & 0.15 & 1.76 & 0.33 & 0.23 & 446.36 \\
\hline \multicolumn{2}{|c|}{ C.D. $1 \%$} & 0.93 & 1.87 & 16.59 & 1.73 & 6.27 & 0.22 & 2.56 & 0.49 & 0.33 & 649.48 \\
\hline
\end{tabular}

The hybrid IIHR $2349 \times$ Arka Saurabh, had higher TSS, pH, lycopene, titratable acidity, calcium, magnesium, and shelf life. Similarly, IIHR $2349 \times$ Arka Vikas recorded higher titratable acidity, pericarp thickness, number of clusters, and yield/plant. Superior performance for fruit diameter, weight, plant height, and number of clusters was exhibited by IIHR $2358 \times$ Arka Ahuti. The IIHR $2357 \times$ Arka Saurabh was superior for magnesium, number of branches, number of clusters, and number of fruits.

\subsection{Heterosis for fruit biochemical, morpho-physiological, and yield attributing traits in tomato hybrids}

The mid parent heterosis (MPH), better parent (BPH), and standard heterosis (SH) of nine hybrids for twenty fruit biochemical, morpho-physiological, and yield characters were estimated (Table II). The MPH, BPH and SH for TSS ranged from -27.42 to $-7.81 \%,-29.69$ to $-7.81 \%$, and -28.33 to $-1.67 \%$ respectively. For pH, the crosses IIHR $2357 \times$ Arka Ahuti, IIHR $2357 \times$ Arka Vikas and IIHR $2358 \times$ Arka Saurabh exhibited higher significant negative MPH (-6.28 to $2.27 \%)$ and BPH (-6.45 to $0.18 \%)$. Similarly, all the crosses exhibited significant negative SH (-9.65 to $-3.33 \%)$. For lycopene, five crosses exhibited positive significant MPH and BPH with the highest in IIHR $2349 \times$ Arka Ahuti (192.08\% MPH and 140.37\% BPH). The crosses IIHR $2349 \times$ Arka Saurabh (128.36\%), IIHR $2357 \times$ Arka Vikas (109.95\%), and IIHR $2357 \times$ Arka Saurabh (60.70\%) exhibited higher positive significant SH.

For ascorbic acid, significant higher positive MPH (38.08\%) and BPH (26.48\%) recorded in IIHR $2357 \times$ Arka Saurabh and significant positive SH (28.82\%) in IIHR $2349 \times$ Arka Ahuti. For titratable acidity, the cross IIHR $2358 \times$ Arka Saurabh recorded higher MPH (17.03\%), BPH (0.10\%), and SH (25.23\%). The crosses IIHR $2349 \times$ Arka Vikas, IIHR $2349 \times$ Arka Saurabh, IIHR $2358 \times$ Arka Vikas and IIHR $2358 \times$ Arka Saurabh exhibited higher positive SH (25.23\%) which is on 
par in crosses. For calcium, the cross IIHR $2349 \times$ Arka Saurabh exhibited higher significant positive MPH (31.75\%) and BPH (11.32\%). For magnesium, six crosses exhibited positive significant MPH with highest in IIHR $2358 \times$ Arka Ahuti (38.54\%), five crosses exhibited higher significant positive BPH with highest in IIHR $2349 \times$ Arka Saurabh (23.28\%). The crosses IIHR $2349 \times$ Arka Saurabh (154.14\%), IIHR $2357 \times$ Arka Saurabh (103.43\%), IIHR $2358 \times$ Arka Saurabh (97.00\%), IIHR $2358 \times$ Arka Ahuti (86.14\%), IIHR $2357 \times$ Arka Ahuti (82.14\%) and IIHR $2358 \times$ Arka Vikas (56.14\%) recorded higher significant positive $\mathrm{SH}$.

Table Il: Heterosis for fruit biochemical, morpho-physiological and yield traits in tomato hybrids

\begin{tabular}{|c|c|c|c|c|c|c|c|c|c|c|}
\hline \multirow[t]{2}{*}{ SI. No. } & \multirow[t]{2}{*}{ Hybrids } & \multicolumn{3}{|l|}{ TSS } & \multicolumn{3}{|l|}{$\mathrm{pH}$} & \multicolumn{3}{|l|}{ Lycopene } \\
\hline & & MPH & BPH & $\mathrm{SH}$ & MPH & $\mathrm{BPH}$ & SH & MPH & $\mathrm{BPH}$ & SH \\
\hline 1 & IIHR 2349 x Arka Vikas & -12.28 & $-21.88^{\star \star}$ & $-16.67 *$ & 0.6 & -1.63 & $-4.74 * \star$ & $23.52^{\star \star}$ & $-18.27 \star \star$ & 1.24 \\
\hline 2 & IIHR 2349 x Arka Ahuti & $-16.67^{\star}$ & $-21.88^{\star \star}$ & $-16.67 *$ & 0.56 & -1.37 & $-5.09 * \star$ & $192.08 * \star$ & $140.37 \star \star$ & -3.73 \\
\hline 3 & IIHR 2349 x Arka Saurabh & -7.81 & -7.81 & -1.67 & 2.27 & 0.18 & $-3.33^{*}$ & $168.81^{\star *}$ & $75.86^{\star \star}$ & $128.36^{\star *}$ \\
\hline 4 & IIHR 2358 x Arka Vikas & $-23.89 * *$ & $-31.75^{\star \star}$ & $-28.33^{\star \star}$ & -0.45 & -0.72 & $-3.33^{*}$ & $-15.42^{\star \star}$ & $-16.27 * \star$ & 3.73 \\
\hline 5 & IIHR 2358 x Arka Ahuti & $-22.69 * \star$ & $-26.98^{\star \star}$ & $-23.33^{\star \star}$ & -1.68 & -2.25 & $-4.82^{\star \star}$ & $16.89 *$ & $-29.10 * \star$ & $-13.93^{*}$ \\
\hline 6 & IIHR 2358 x Arka Saurabh & -8.66 & -9.38 & -3.33 & $-2.62^{*}$ & $-3.06^{\star}$ & -5.61 *夫 & $-63.96 * \star$ & $-65.13^{\star \star}$ & $-54.73^{\star *}$ \\
\hline 7 & IIHR 2357 x Arka Vikas & -12.73 & $-20.00 *$ & $-20.00 *$ & $-2.68^{*}$ & $-2.81^{\star}$ & $-5.88^{\star \star}$ & $37.46^{\star \star}$ & $15.62^{\star \star}$ & $109.95^{\star *}$ \\
\hline 8 & IIHR 2357 x Arka Ahuti & $-15.52^{*}$ & $-18.33^{*}$ & $-18.33^{*}$ & $-6.28 * \star$ & $-6.45^{\star \star}$ & $-9.65^{\star \star}$ & $-40.05^{\star \star}$ & $-65.75^{\star \star}$ & $-37.81^{\star *}$ \\
\hline 9 & IIHR 2357 x Arka Saurabh & $-27.42^{\star \star}$ & $-29.69 * \star$ & $-25.00 * \star$ & -1.04 & -1.09 & $-4.47 \star \star$ & 3.19 & $-11.51^{\star \star}$ & $60.70 \star *$ \\
\hline \multirow[t]{2}{*}{ Sl. No. } & Hybrids & \multicolumn{3}{|c|}{ Ascorbic acid } & \multicolumn{3}{|c|}{ Titratable acidity } & \multicolumn{3}{|l|}{ Calcium } \\
\hline & & MPH & BPH & $\mathrm{SH}$ & MPH & BPH & $\mathrm{SH}$ & MPH & BPH & $\mathrm{SH}$ \\
\hline 1 & IIHR 2349 x Arka Vikas & $-30.74 * \star$ & $-55.00 * \star$ & $-21.52^{\star \star}$ & -16.51 & -19.76 & 25.23 & $-17.32^{\star \star}$ & $-30.14^{\star \star}$ & $-41.65^{\star *}$ \\
\hline 2 & IIHR 2349 x Arka Ahuti & $-15.18^{\star \star}$ & $-26.13^{\star \star}$ & $28.82^{\star \star}$ & -7.14 & $-29.94 *$ & 9.35 & $-57.64^{\star \star}$ & $-63.88^{\star *}$ & $-70.49 * \star$ \\
\hline 3 & IIHR 2349 x Arka Saurabh & $-54.53^{\star \star}$ & $-68.91 * \star$ & $-45.79 * \star$ & 2.29 & -19.76 & 25.23 & $31.75^{\star \star}$ & $11.32^{\star \star}$ & $-7.03^{\star \star}$ \\
\hline 4 & IIHR 2358 x Arka Vikas & 0.70 & 0.35 & $-47.61^{\star \star}$ & -6.94 & -12.99 & 25.23 & $-5.61 * \star$ & $-14.67 * \star$ & $-28.73^{\star *}$ \\
\hline 5 & IIHR 2358 x Arka Ahuti & $15.80 * \star$ & $-18.89 \star \star$ & 4.93 & -2.28 & -20.15 & 0.10 & $1.35^{\star \star}$ & $-7.46^{\star \star}$ & $-24.39 * *$ \\
\hline 6 & IIHR 2358 x Arka Saurabh & -7.15 & $-16.00 \star \star$ & $-46.19 * \star$ & 17.03 & 0.10 & 25.23 & $-19.78^{\star \star}$ & $-27.48 * \star$ & $-39.43^{\star \star}$ \\
\hline 7 & IIHR 2357 x Arka Vikas & 2.07 & 1.03 & $-46.15^{\star \star}$ & $-72.76^{\star \star}$ & $-73.38 * \star$ & $-61.68^{\star \star}$ & $-36.74^{\star \star}$ & $-41.34 * \star$ & $-51.01 * \star$ \\
\hline 8 & IIHR 2357 x Arka Ahuti & $9.85^{\star *}$ & $-22.45^{\star \star}$ & 0.33 & -6.90 & $-26.53^{*}$ & 0.93 & $-4.12^{\star \star}$ & $-10.18^{\star \star}$ & -26.61 ** \\
\hline 9 & IIHR 2357 x Arka Saurabh & $38.08^{\star *}$ & $26.48^{\star \star}$ & $-18.97 \star \star$ & $-58.68 \star \star$ & $-65.99 * *$ & $-53.27 \star \star$ & $-3.97 \star \star$ & $-10.95^{\star \star}$ & $-25.63^{\star *}$ \\
\hline \multirow[t]{2}{*}{ SI. No. } & \multirow[t]{2}{*}{ Hybrids } & \multicolumn{3}{|c|}{ Magnesium } & \multicolumn{3}{|c|}{ Fruit length } & \multicolumn{3}{|c|}{ Fruit diameter } \\
\hline & & MPH & BPH & $\mathrm{SH}$ & MPH & $\mathrm{BPH}$ & $\mathrm{SH}$ & MPH & $\mathrm{BPH}$ & SH \\
\hline 1 & IIHR 2349 x Arka Vikas & $-61.76^{\star \star}$ & $-63.34 \star \star$ & $-24.43^{\star \star}$ & 4.87 & -3.59 & $-20.61^{\star \star}$ & 9.24 & 7.41 & -1.12 \\
\hline 2 & IIHR 2349 x Arka Ahuti & $-59.30 \star \star$ & $-64.52^{\star \star}$ & $-26.86 * \star$ & 7.89 & 3.39 & $-28.61 \star \star$ & $18.22^{\star \star}$ & 7.65 & -0.91 \\
\hline 3 & IIHR 2349 x Arka Saurabh & $28.59 * *$ & $23.28^{\star \star}$ & $154.14^{\star \star}$ & 27.27 ** & $21.24^{\star \star}$ & -7.51 & $13.83^{*}$ & 7.08 & -1.43 \\
\hline 4 & IIHR 2358 x Arka Vikas & $2.53^{\star *}$ & $-17.45^{\star \star}$ & $56.14^{\star *}$ & $-17.47^{\star \star}$ & -7.39 & $-23.73^{\star \star}$ & 2.24 & -0.48 & $-11.45^{\star}$ \\
\hline 5 & IIHR 2358 x Arka Ahuti & $38.54^{\star \star}$ & $21.44^{\star \star}$ & $86.14^{\star *}$ & 6.68 & $39.70 \star \star$ & $-11.58^{*}$ & $31.76^{\star *}$ & $25.01^{\star \star}$ & 5.30 \\
\hline 6 & IIHR 2358 x Arka Saurabh & $29.36^{* *}$ & $4.15^{\star \star}$ & $97.00 * *$ & 4.15 & $22.03^{\star \star}$ & -6.91 & $14.66^{*}$ & $12.55^{\star}$ & -5.20 \\
\hline 7 & IIHR 2357 x Arka Vikas & $-46.25^{\star \star}$ & $-50.53^{\star \star}$ & $-6.43^{\star \star}$ & -8.34 & -8.34 & $-24.52 \star \star$ & 16.02 ** & $13.14 *$ & 0.67 \\
\hline 8 & IIHR 2357 x Arka Ahuti & $16.65^{\star \star}$ & $14.56^{\star \star}$ & $82.14^{\star \star}$ & $16.53^{\star *}$ & $34.07 * \star$ & $-15.14 \star \star$ & $25.32^{\star \star}$ & $18.67 \star \star$ & 0.35 \\
\hline 9 & IIHR 2357 x Arka Saurabh & $16.86^{\star \star}$ & $7.55^{\star \star}$ & $103.43^{\star *}$ & -9.02 & -5.40 & $-27.84^{\star \star}$ & $13.56^{*}$ & 11.26 & -5.92 \\
\hline
\end{tabular}

Cont'd...( Table II: Heterosis for fruit biochemical, morpho-physiological and yield traits in tomato hybrids) 


\begin{tabular}{|c|c|c|c|c|c|c|c|c|c|c|}
\hline \multirow[t]{2}{*}{ SI. No. } & \multirow[t]{2}{*}{ Hybrids } & \multicolumn{3}{|c|}{ Fruit weight } & \multicolumn{3}{|c|}{ Fruit firmness } & \multicolumn{3}{|c|}{ Pericarp thickness } \\
\hline & & MPH & BPH & $\mathrm{SH}$ & MPH & BPH & SH & MPH & BPH & $\mathrm{SH}$ \\
\hline 1 & IIHR 2349 x Arka Vikas & 27.31 ** & $22.42^{\star}$ & -11.87 & $40.73^{*}$ & 29.03 & -13.62 & $30.93^{*}$ & 29.28 & -5.06 \\
\hline 2 & IIHR 2349 x Arka Ahuti & $36.72^{\star \star}$ & 3.52 & $-25.47 * \star$ & -1.20 & -17.00 & $-31.89 * *$ & 0.44 & -6.32 & $-22.51 *$ \\
\hline 3 & IIHR 2349 x Arka Saurabh & $48.79 * \star$ & $28.35^{\star \star}$ & -7.59 & 9.19 & -2.35 & $-30.90 *$ & 16.68 & 13.62 & -14.14 \\
\hline 4 & IIHR 2358 x Arka Vikas & -12.90 & -15.63 & $-40.19 * \star$ & $30.10 *$ & 26.55 & -15.28 & 3.18 & -1.26 & -20.66 \\
\hline 5 & IIHR 2358 x Arka Ahuti & $89.15^{\star \star}$ & $43.97 \star \star$ & 2.06 & 6.06 & -6.07 & $-22.92^{\star}$ & 4.45 & 2.96 & -14.84 \\
\hline 6 & IIHR 2358 x Arka Saurabh & $29.56^{\star \star}$ & 12.50 & $-20.25^{\star \star}$ & 12.02 & 6.10 & $-24.92^{\star}$ & $-24.39 *$ & -26.65 & $-41.06^{\star *}$ \\
\hline 7 & IIHR 2357 x Arka Vikas & 13.50 & 7.29 & $-19.94 * \star$ & $37.42^{\star}$ & $33.50 *$ & -10.63 & 16.45 & 9.30 & -19.73 \\
\hline 8 & IIHR 2357 x Arka Ahuti & $75.03 * \star$ & $30.94^{\star \star}$ & -2.29 & 2.97 & -8.91 & $-25.25^{\star}$ & 4.30 & -7.24 & $-23.27 *$ \\
\hline 9 & IIHR 2357 x Arka Saurabh & 2.79 & -12.64 & -34.81 ** & -12.16 & -16.90 & $-41.20 * \star$ & 10.60 & 2.46 & $-22.60 *$ \\
\hline \multirow[t]{2}{*}{ SI. No. } & Hybrids & \multicolumn{3}{|c|}{ Pulp content } & \multicolumn{3}{|c|}{ Locule number } & \multicolumn{3}{|l|}{ Shelf life } \\
\hline & & MPH & BPH & $\mathrm{SH}$ & MPH & BPH & $\mathrm{SH}$ & $\mathrm{MPH}$ & BPH & $\mathrm{SH}$ \\
\hline 1 & IIHR 2349 x Arka Vikas & 0.03 & -9.46 & -9.46 & -9.46 & 4.88 & 6.24 & 21.53 & 4.88 & 6.24 \\
\hline 2 & IIHR 2349 x Arka Ahuti & -4.05 & -11.41 & -11.41 & -11.41 & $-25.83^{\star}$ & -13.55 & -9.05 & $-25.83^{\star}$ & -13.55 \\
\hline 3 & IIHR 2349 x Arka Saurabh & 2.80 & -3.45 & -3.45 & -3.45 & 11.15 & $26.45^{\star}$ & $35.02^{\star \star}$ & 11.15 & $26.45^{\star}$ \\
\hline 4 & IIHR 2358 x Arka Vikas & 2.38 & 20.00 & 20.00 & 20.00 & -8.70 & -7.53 & 8.04 & -8.70 & -7.53 \\
\hline 5 & IIHR 2358 x Arka Ahuti & -4.90 & 15.52 & 15.52 & 15.52 & -16.61 & -2.80 & 4.27 & -16.61 & -2.80 \\
\hline 6 & IIHR 2358 x Arka Saurabh & 0.36 & 30.36 & 30.36 & 30.36 & 0.57 & 14.41 & $24.59 *$ & 0.57 & 14.41 \\
\hline 7 & IIHR 2357 x Arka Vikas & 1.09 & -4.29 & -4.29 & -4.29 & 18.90 & 20.43 & $37.09 \star \star$ & 18.90 & 20.43 \\
\hline 8 & IIHR 2357 x Arka Ahuti & -3.34 & -4.96 & -4.96 & -4.96 & -15.87 & -1.94 & 2.70 & -15.87 & -1.94 \\
\hline 9 & IIHR 2357 x Arka Saurabh & -0.94 & -12.41 & -12.41 & -12.41 & -8.51 & 4.09 & 10.63 & -8.51 & 4.09 \\
\hline \multirow[t]{2}{*}{ SI. No. } & \multirow[t]{2}{*}{ Hybrids } & \multicolumn{3}{|c|}{ Plant height } & \multicolumn{3}{|c|}{ Number of branches } & \multicolumn{3}{|c|}{ Number of clusters } \\
\hline & & MPH & $\mathrm{BPH}$ & $\mathrm{SH}$ & MPH & $\mathrm{BPH}$ & $\mathrm{SH}$ & MPH & $\mathrm{BPH}$ & $\mathrm{SH}$ \\
\hline 1 & IIHR 2349 x Arka Vikas & 10.05 & 4.05 & -10.58 & -14.61 & -17.39 & -5.00 & $-13.70^{\star \star}$ & $-6.36^{\star \star}$ & $8.87^{\star \star *}$ \\
\hline 2 & IIHR 2349 x Arka Ahuti & 21.41 & 14.37 & -0.94 & 11.63 & 11.63 & 20.00 & $-13.78^{\star \star}$ & $-17.80 * \star$ & $-4.43^{\star}$ \\
\hline 3 & IIHR 2349 x Arka Saurabh & -0.54 & -10.60 & -14.19 & -3.53 & -4.65 & 2.50 & $4.10 *$ & $-13.98 * \star$ & 0 \\
\hline 4 & IIHR 2358 x Arka Vikas & -3.21 & -8.41 & -21.29 & -6.12 & -11.54 & 15.00 & $-3.56^{\star}$ & $-21.08^{\star \star}$ & -13.30 ** \\
\hline 5 & IIHR 2358 x Arka Ahuti & 36.07 & 28.28 & $11.11^{\star \star}$ & -24.21 & -30.77 & -10.00 & $26.40 * \star$ & $5.14 \star \star$ & $10.84^{\star *}$ \\
\hline 6 & IIHR 2358 x Arka Saurabh & 5.27 & -5.30 & -9.10 & -4.26 & -13.46 & 12.50 & $31.08^{\star \star}$ & $25.97 \star \star$ & $-4.43^{\star}$ \\
\hline 7 & IIHR 2357 x Arka Vikas & -4.71 & -10.28 & -22.89 & -4.55 & -8.70 & 5.00 & $-25.20 * \star$ & $-36.77^{\star \star}$ & $-30.54^{\star *}$ \\
\hline 8 & IIHR 2357 x Arka Ahuti & 26.44 & 18.62 & $2.74 * \star$ & $-42.35^{\star}$ & $-43.02^{\star}$ & -38.75 & $19.02^{\star \star}$ & 2.34 & $7.88^{\star \star}$ \\
\hline 9 & IIHR 2357 x Arka Saurabh & 13.08 & 1.26 & -2.81 & 21.43 & 21.43 & 27.50 & $40.91^{\star \star}$ & $40.91 \star \star$ & $6.90 * \star$ \\
\hline
\end{tabular}

Cont’d...( Table Il: Heterosis for fruit biochemical, morpho-physiological and yield traits in tomato hybrids) 


\begin{tabular}{|c|c|c|c|c|c|c|c|}
\hline \multirow[t]{2}{*}{ SI. No. } & \multirow[t]{2}{*}{ Hybrids } & \multicolumn{3}{|c|}{ Number of fruit/cluster } & \multicolumn{3}{|c|}{ Yield/plant } \\
\hline & & MPH & BPH & $\mathrm{SH}$ & MPH & BPH & $\mathrm{SH}$ \\
\hline 1 & IIHR 2349 x Arka Vikas & 6.41 & $-5.68^{\star}$ & $-27.83^{\star \star}$ & $140.23^{\star \star}$ & $83.12^{\star \star}$ & 52.27 ** \\
\hline 2 & IIHR 2349 x Arka Ahuti & $32.05^{\star \star}$ & $17.05^{\star \star}$ & $-10.43^{\star *}$ & 30.59 & 3.03 & -14.33 \\
\hline 3 & IIHR 2349 x Arka Saurabh & $11.54^{\star \star}$ & -1.14 & $-24.35^{\star \star}$ & $40.59 *$ & 20.90 & 0.53 \\
\hline 4 & IIHR 2358 x Arka Vikas & $27.94^{\star \star}$ & $27.94^{\star \star}$ & $-24.35^{\star \star}$ & 17.40 & -6.99 & -30.59 \\
\hline 5 & IIHR 2358 x Arka Ahuti & $51.47^{* \star}$ & $51.47^{* \star}$ & $-10.43^{\star *}$ & 30.19 & 7.02 & -20.14 \\
\hline 6 & IIHR 2358 x Arka Saurabh & $-7.35^{\star}$ & $-7.35^{\star}$ & $-45.22 \star \star$ & $71.81 * \star$ & $54.82^{\star}$ & 15.53 \\
\hline 7 & IIHR 2357 x Arka Vikas & $5.88^{\star}$ & 5.88 & $-37.39 * \star$ & 36.86 & 9.66 & -20.61 \\
\hline 8 & IIHR 2357 x Arka Ahuti & $51.47^{\star \star}$ & $51.47 * \star$ & $-10.43^{\star \star}$ & 25.62 & 4.50 & -24.34 \\
\hline 9 & IIHR 2357 x Arka Saurabh & $66.18 * \star$ & $66.18 * \star$ & -1.74 & $76.04 * \star$ & $60.80 *$ & 16.41 \\
\hline
\end{tabular}

For fruit length, IIHR $2349 \times$ Arka Saurabh (27.27\%) and IIHR $2357 \times$ Arka Ahuti (16.53\%) were exhibited higher significant positive MPH. Four crosses recorded significant positive BPH which was highest in IIHR $2358 \times$ Arka Ahuti (39.70\%). For fruit diameter, six crosses exhibited positive significant MPH which was highest in the cross IIHR $2358 \times$ Arka Ahuti (31.76\%) and four crosses exhibited higher significant positive BPH with highest in IIHR $2358 \times$ Arka Ahuti (25.01\%). For fruit weight, six crosses exhibited positive significant MPH with the highest in IIHR $2358 \times$ Arka Ahuti (89.15\%). IIHR $2358 \times$ Arka Ahuti (43.97\%) cross recorded the highest significant positive BPH out of four crosses. For fruit firmness, three crosses exhibited positive significant MPH with highest by the cross IIHR $2349 \times$ Arka Vikas (40.73\%). The cross IIHR $2357 \times$ Arka Vikas exhibited higher significant positive $\mathrm{BPH}(33.50 \%)$.

For pericarp thickness, the highest MPH and BPH recorded in IIHR $2349 \times$ Arka Vikas (30.93\%) and IIHR $2349 \times$ Arka Vikas (29.28\%) respectively. The cross IIHR $2349 \times$ Arka Saurabh exhibited higher MPH (2.80\%) and the cross IIHR $2358 \times$ Arka Vikas recorded higher positive BPH (3.10\%) and SH (1.47\%) for pulp content. For locule number, the cross IIHR $2357 \times$ Arka Saurabh exhibited higher negative heterosis MPH (-12.41\%). IIHR $2349 \times$ Arka Ahuti recorded the highest significant negative BPH (-25.83\%) and the cross IIHR $2349 \times$ Arka Ahuti recorded negative SH (-13.55\%). For shelf life, the crosses IIHR $2357 \times$ Arka Vikas (37.09\%), IIHR $2349 \times$ Arka Saurabh (35.02\%), and IIHR $2358 \times$ Arka Saurabh (24.59\%) exhibited higher significant positive MPH. The crosses IIHR $2357 \times$ Arka Vikas and IIHR $2349 \times$ Arka Saurabh exhibited higher positive BPH (18.90\%) and SH (26.45\%) respectively.

For Plant height, the cross IIHR $2358 \times$ Arka Ahuti exhibited higher positive MPH (36.07\%) and the cross IIHR $2358 \times$ Arka Ahuti recorded the highest positive BPH (28.28\%). The crosses IIHR $2358 \times$ Arka Ahuti (11.11\%) and IIHR $2357 \times$ Arka Ahuti (2.74\%) were recorded significant positive SH. For number of branches, the cross IIHR $2357 \times$ Arka Saurabh exhibited higher positive MPH (21.43\%), BPH (21.43\%), and SH (27.50\%). For number of clusters, the cross IIHR $2357 \times$ Arka Saurabh exhibited higher positive MPH (40.91\%) and BPH (40.91\%). Four crosses recorded higher significant positive SH with the highest in the cross IIHR $2358 \times$ Arka Ahuti (10.84\%) followed by IIHR $2349 \times$ Arka Vikas (8.87\%), IIHR $2357 \times$ Arka Ahuti $(7.88 \%)$, and IIHR $2357 \times$ Arka Saurabh (6.90\%).

For number of fruit/cluster, seven crosses exhibited positive significant MPH with the highest in the cross IIHR $2357 \times$ Arka Saurabh $(66.18 \%)$. Five crosses exhibited higher significant positive BPH with the highest in the cross IIHR $2357 \times$ Arka Saurabh (66.18\%) followed by IIHR $2357 \times$ Arka Ahuti (51.47\%), IIHR $2358 \times$ Arka Ahuti (51.47\%), IIHR $2358 \times$ Arka Vikas (27.94\%) and IIHR $2349 \times$ Arka Ahuti (17.05\%). For yield/plant, four crosses IIHR $2349 \times$ Arka Vikas (140.23\%), IIHR $2357 \times$ Arka Saurabh (76.04\%), IIHR $2358 \times$ Arka Saurabh (71.81\%), and IIHR $2349 \times$ Arka Saurabh (40.59\%) recorded the highest significant positive MPH. Three crosses IIHR $2349 \times$ Arka Vikas (83.12\%), IIHR $2357 \times$ Arka Saurabh (60.80\%), and IIHR $2358 \times$ Arka Saurabh (54.82\%) recorded the highest significant positive BPH and the cross IIHR $2349 \times$ Arka Vikas (52.27\%) exhibited higher significant positive SH.

\subsection{ANOVA for fruit biochemical, morpho-physiological and yield attributing traits}

A significant difference was observed among the genotypes for all the characters except fruit firmness, pericarp thickness, pulp content, locule number, and number of branches (Table III). Parents differed significantly for all the characters except for fruit firmness, pericarp thickness, pulp content, locule number, number of branches and yield/plant. A significant difference was observed among the lines for $\mathrm{pH}$, lycopene, ascorbic acid, calcium, magnesium, fruit length, locule number, plant height, number of cluster and number of fruit/cluster. Testers exhibited significant differences for TSS, lycopene, ascorbic acid, titratable acidity, calcium, magnesium, fruit length, diameter, weight and number of clusters.

Table III: Analysis of variance for fruit biochemical, morpho-physiological and yield attributing traits 


\begin{tabular}{|c|c|c|c|c|c|c|c|c|c|c|c|}
\hline \multirow{2}{*}{$\begin{array}{l}\text { Source of } \\
\text { variation }\end{array}$} & \multirow[t]{2}{*}{ Df } & \multicolumn{10}{|c|}{ Mean sum of squares } \\
\hline & & TSS & $\mathrm{pH}$ & Lycopene & $\begin{array}{l}\text { Ascorbic } \\
\text { acid }\end{array}$ & $\begin{array}{l}\text { Titratable } \\
\text { acidity }\end{array}$ & Calcium & Magnesium & $\begin{array}{l}\text { Fruit } \\
\text { length }\end{array}$ & $\begin{array}{l}\text { Fruit } \\
\text { diameter }\end{array}$ & Fruit weight \\
\hline Replication & 1 & 0.04 & 0.01 & 0.18 & 0.01 & 0.01 & 0.01 & 0.01 & 12.31 & 0.01 & 0.02 \\
\hline Genotypes & 14 & 0.26 ** & $0.02^{\star *}$ & $2.97^{\star \star}$ & $53.73^{\star \star}$ & 0.06 ** & $18.91^{\star \star}$ & $26.72^{\star \star}$ & $66.87^{\star \star}$ & $29.82^{\star \star}$ & $236.74^{\star \star}$ \\
\hline Parents & 5 & $0.16^{*}$ & $0.02^{*}$ & $2.84^{\star \star}$ & $101.72^{\star \star}$ & $0.05^{\star \star}$ & $8.35^{\star \star}$ & 10.60 ** & $114.45^{\star \star}$ & $14.06^{*}$ & $172.10 * \star$ \\
\hline Lines & 2 & 0.02 & $0.04 * \star$ & $4.08^{\star \star}$ & $185.84^{\star \star}$ & 0.01 & $3.76 \star \star$ & $20.17 \star \star$ & $175.01^{\star \star}$ & 8.20 & 2.94 \\
\hline Testers & 2 & $0.25^{\star}$ & 0.01 & $2.75^{\star \star}$ & $64.86 * \star$ & $0.07 * \star$ & $0.18^{\star \star}$ & $4.20 * \star$ & $58.54^{\star \star}$ & $18.94^{*}$ & $173.04^{\star *}$ \\
\hline $\begin{array}{l}\text { Lines } x \\
\text { Testers }\end{array}$ & 1 & $0.24^{*}$ & 0.01 & 0.54 ** & $7.19 * \star$ & 0.11 ** & $34.07^{\star *}$ & $4.25^{\star \star}$ & $105.14^{\star \star}$ & 16.03 & $508.56^{\star \star}$ \\
\hline $\begin{array}{l}\text { Parents x } \\
\text { Hybrids }\end{array}$ & 1 & $1.70 * *$ & $0.04^{*}$ & $0.92 * \star$ & $10.66^{\star *}$ & $0.10 * *$ & $22.83^{\star \star}$ & $4.09 * *$ & 10.80 & $270.48^{\star \star}$ & $1148.32 * *$ \\
\hline Hybrids & 8 & $0.15^{\star}$ & $0.02^{\star \star}$ & $3.31^{\star \star}$ & $29.12^{\star \star}$ & $0.07 * \star$ & $25.01^{\star \star}$ & $39.63^{\star \star}$ & $44.14^{\star \star}$ & 9.58 & $163.18^{* *}$ \\
\hline Error & 14 & 0.04 & 0.01 & 0.01 & 0.12 & 0.01 & 0.01 & 0.01 & 4.29 & 3.74 & 12.33 \\
\hline \multirow{2}{*}{$\begin{array}{l}\text { Source of } \\
\text { variation }\end{array}$} & \multirow[t]{2}{*}{ Df } & \multicolumn{10}{|c|}{ Mean sum of squares } \\
\hline & & $\begin{array}{l}\text { Fruit } \\
\text { firmness }\end{array}$ & $\begin{array}{l}\text { Pericarp } \\
\text { thickness }\end{array}$ & $\begin{array}{l}\text { Pulp } \\
\text { content }\end{array}$ & $\begin{array}{l}\text { Locule } \\
\text { number }\end{array}$ & $\begin{array}{l}\text { Shelf } \\
\text { life }\end{array}$ & $\begin{array}{l}\text { Plant } \\
\text { height }\end{array}$ & $\begin{array}{l}\text { No. of } \\
\text { branches }\end{array}$ & $\begin{array}{l}\text { No. of } \\
\text { clusters }\end{array}$ & $\begin{array}{l}\text { No. of } \\
\text { fruit/cluster }\end{array}$ & Yield/plant \\
\hline Replication & 1 & 0.01 & 0.38 & 76.10 & 0.04 & 1.88 & 0.01 & 3.68 & 0.04 & 0.01 & 264538.82 \\
\hline Genotypes & 14 & 0.19 & 0.53 & 36.16 & 0.28 & $33.65^{\star *}$ & $0.02^{* *}$ & 0.88 & $5.35^{\star \star}$ & $1.36^{\star *}$ & $304297.27 * \star$ \\
\hline Parents & 5 & 0.14 & 0.30 & 41.84 & 0.67 & $49.75^{\star \star}$ & $0.02 *$ & 0.3 & $8.63^{* \star}$ & $0.33^{\star *}$ & 95470.93 \\
\hline Lines & 2 & 0.03 & 0.45 & 81.60 & $1.48 *$ & 0.62 & $0.04^{\star \star}$ & 0.61 & $13.09 * *$ & $0.67^{\star *}$ & 12443.95 \\
\hline Testers & 2 & 0.11 & 0.17 & 18.59 & 0.02 & 7.15 & 0.01 & 0.09 & $7.04^{\star \star}$ & 0.01 & 27230.86 \\
\hline $\begin{array}{l}\text { Lines } x \\
\text { Testers }\end{array}$ & 1 & $0.43^{*}$ & 0.28 & 8.86 & 0.37 & $233.20 * \star$ & 0.01 & 0.12 & $2.90 * *$ & $0.33^{\star *}$ & 398005.04 ** \\
\hline $\begin{array}{l}\text { Parents x } \\
\text { Hybrids }\end{array}$ & 1 & $0.52^{\star}$ & 0.61 & 2.20 & 0.01 & $71.06^{\star \star}$ & $0.04^{\star}$ & 0.83 & $2.47^{\star \star}$ & $6.57^{\star \star}$ & $1567279.52^{\star \star}$ \\
\hline Hybrids & 8 & 0.17 & 0.66 & 36.86 & 0.06 & $18.91^{\star}$ & $0.02^{\star \star}$ & 1.24 & $3.65^{\star \star}$ & $1.35^{\star \star}$ & $276940.95^{\star \star}$ \\
\hline Error & 14 & 0.08 & 0.31 & 24.45 & 0.26 & 4.91 & 0.01 & 0.58 & 0.02 & 0.01 & 37466.93 \\
\hline
\end{tabular}

Line $\times$ Tester recorded significant differences for all the traits except for $\mathrm{pH}$, fruit diameter, pericarp thickness, pulp content, locule number, plant height and number of branches. The mean sum of squares of parents v/s hybrids was significant for all the characters except fruit length, pericarp thickness, pulp content, locule number and number of branches. Hybrids differed significantly for all the traits except fruit diameter, firmness, pericarp thickness, pulp content, locule number, number of branches.

\subsection{ANOVA for combining ability and Proportional contribution of lines, testers and line $\times$ tester for hybrids performance}

The crosses exhibited high level of significance for all the traits except TSS, fruit firmness, pericarp thickness, pulp content, locule number and number of branches (Table IV). The variance due to crosses was further portioned into variance due to lines, testers and line $\times$ testers. The mean sum of squares due to lines was highly significant for ascorbic acid, fruit firmness and pulp content whereas, mean sum of squares due to testers was significant for pulp content. The Line $\times$ Tester interaction variance was highly significant for lycopene, ascorbic acid, titratable acidity, calcium, magnesium, fruit length, diameter, weight, number of branches, number of clusters and yield/plant. The per cent contribution of lines towards total variation was higher for ascorbic acid followed by fruit firmness and least for titratable acidity. Similarly, the per cent contribution of tester towards total variation was higher for titratable acidity and least for fruit weight.

\subsection{Variance due to combining ability effects}

The variance due to general combining ability was highly significant for ascorbic acid, fruit firmness, and pulp content whereas, variance due to specific combining ability was significant for $\mathrm{pH}$, lycopene, ascorbic acid, titratable acidity, calcium, magnesium, fruit length, weight, plant height, number of clusters, number of fruits/cluster, and yield/plant (Table VI). It was evident that among twenty studied traits most manifested a higher degree of SCA variance as compared to GCA variance. The GCA/SCA ratio was less than one for all the characters except for pH, ascorbic acid, fruit firmness, and plant height.

Table IV: Analysis of variance for combining ability in lines and testers for fruit biochemical, morpho-physiological and yield attributing traits 


\begin{tabular}{|c|c|c|c|c|c|c|c|c|c|c|c|}
\hline \multirow{2}{*}{$\begin{array}{l}\text { Source of } \\
\text { variation }\end{array}$} & \multirow[t]{2}{*}{ Df } & \multicolumn{10}{|c|}{ Mean sum of squares } \\
\hline & & TSS & $\mathrm{pH}$ & Lycopene & $\begin{array}{l}\text { Ascorbic } \\
\text { acid }\end{array}$ & $\begin{array}{l}\text { Titratable } \\
\text { acidity }\end{array}$ & Calcium & Magnesium & $\begin{array}{l}\text { Fruit } \\
\text { length }\end{array}$ & $\begin{array}{l}\text { Fruit } \\
\text { diameter }\end{array}$ & Fruit weight \\
\hline Replications & 1 & 0.02 & 0.01 & 0.06 & 0.29 & 0.013 & 0.01 & 0.01 & 2.98 & 6.94 & 0.01 \\
\hline Crosses & 8 & 0.15 & $0.02^{\star}$ & $3.31^{\star *}$ & $29.12^{\star}$ & $0.06^{* *}$ & $25.01^{\star \star}$ & $39.63^{\star \star}$ & $44.14^{\star \star}$ & $9.58^{* *}$ & $163.18 * \star$ \\
\hline Line effect & 2 & 0.21 & 0.03 & 2.94 & $90.48^{*}$ & 0.002 & 20.26 & 91.11 & 36.44 & 13.43 & 159.51 \\
\hline $\begin{array}{l}\text { Tester } \\
\text { effect }\end{array}$ & 2 & 0.13 & 0.03 & 3.39 & 7.95 & 0.18 & 4.47 & 15.27 & 33.12 & 2.47 & 14.61 \\
\hline $\begin{array}{l}\text { Line } x \\
\text { Tester } \\
\text { effect }\end{array}$ & 4 & 0.13 & 0.02 & $3.45^{\star \star}$ & $9.02 * \star$ & $0.04 * \star$ & $37.65^{\star \star}$ & $26.07 * \star$ & $53.51^{\star \star}$ & 11.21 ** & $239.30 * \star$ \\
\hline Error & 8 & 0.07 & 0.01 & 0.01 & 0.03 & 0.01 & 0.01 & 0.01 & 4.76 & 1.00 & 9.01 \\
\hline Total & 17 & 0.10 & 0.01 & 1.56 & 13.74 & 0.03 & 11.77 & 18.65 & 23.19 & 5.39 & 81.03 \\
\hline \multirow{2}{*}{$\begin{array}{l}\text { Source of } \\
\text { variation }\end{array}$} & \multirow[t]{2}{*}{ Df } & \multicolumn{10}{|c|}{ Mean sum of squares } \\
\hline & & $\begin{array}{l}\text { Fruit } \\
\text { firmness }\end{array}$ & $\begin{array}{l}\text { Pericarp } \\
\text { thickness }\end{array}$ & $\begin{array}{l}\text { Pulp } \\
\text { content }\end{array}$ & $\begin{array}{l}\text { Locule } \\
\text { number }\end{array}$ & Shelf life & $\begin{array}{l}\text { Plant } \\
\text { height }\end{array}$ & $\begin{array}{l}\text { No. of } \\
\text { branches }\end{array}$ & $\begin{array}{l}\text { No. of } \\
\text { clusters }\end{array}$ & $\begin{array}{l}\text { No. of } \\
\text { fruits/cluster }\end{array}$ & Yield/plant \\
\hline Replications & 1 & 0.03 & 0.69 & 118.42 & 0.22 & 1.50 & 0.01 & 6.13 & 0.01 & 0.01 & 507460.90 \\
\hline Crosses & 8 & 0.17 & 0.66 & 36.86 & 0.06 & $18.90^{*}$ & $0.02 *$ & 1.24 & $3.65^{\star *}$ & $1.35^{\star \star}$ & $276940.90 * *$ \\
\hline Line effect & 2 & $0.52^{\star}$ & 0.62 & $86.82^{\star \star}$ & 0.01 & 36.42 & 0.02 & 1.38 & 4.54 & 1.96 & 276918.80 \\
\hline $\begin{array}{l}\text { Tester } \\
\text { effect }\end{array}$ & 2 & 0.03 & 0.74 & $53.96^{*}$ & 0.09 & 3.48 & 0.03 & 0.20 & 0.70 & 0.51 & 213763.80 \\
\hline $\begin{array}{l}\text { Line } x \\
\text { Tester } \\
\text { effect }\end{array}$ & 4 & 0.06 & 0.64 & 3.32 & 0.07 & 17.85 & 0.02 & $1.69 *$ & $4.68^{\star *}$ & 1.46 & 308540.60 ** \\
\hline Error & 8 & 0.07 & 0.33 & 18.31 & 0.08 & 4.95 & 0.01 & 0.38 & 0.02 & 0.01 & 34160.18 \\
\hline Total & 17 & 0.12 & 0.51 & 32.93 & 0.08 & 11.32 & 0.01 & 1.12 & 1.73 & 0.64 & 176251.20 \\
\hline
\end{tabular}

Table V: Per cent contribution of lines, testers and line $x$ tester interaction for hybrids performance

\begin{tabular}{|c|c|c|c|c|c|c|c|c|c|}
\hline \multirow{2}{*}{$\begin{array}{l}\text { SI. } \\
\text { No. }\end{array}$} & \multirow[t]{2}{*}{ Characters } & \multicolumn{3}{|c|}{ Percent contribution } & \multirow{2}{*}{$\begin{array}{l}\text { SI. } \\
\text { No. }\end{array}$} & \multirow[t]{2}{*}{ Characters } & \multicolumn{3}{|c|}{ Percent contribution } \\
\hline & & Lines & Testers & $\begin{array}{l}\text { Line } \times \text { Tester } \\
\text { interaction }\end{array}$ & & & Lines & Testers & $\begin{array}{l}\text { Line } \times \text { Tester } \\
\text { interaction }\end{array}$ \\
\hline 1 & TSS & 34.60 & 21.24 & 44.16 & 11 & Fruit firmness & 75.37 & 5.35 & 19.28 \\
\hline 2 & $\mathrm{pH}$ & 27.30 & 33.77 & 38.93 & 12 & Pericarp thickness & 23.32 & 28.18 & 48.50 \\
\hline 3 & Lycopene & 22.22 & 25.66 & 52.13 & 13 & Pulp content & 58.89 & 36.60 & 4.51 \\
\hline 4 & Ascorbic acid & 77.68 & 6.83 & 15.50 & 14 & Locule number & 2.04 & 38.78 & 59.18 \\
\hline 5 & $\begin{array}{l}\text { Titratable } \\
\text { acidity }\end{array}$ & 0.84 & 68.71 & 30.45 & 15 & Shelf life & 48.16 & 4.61 & 47.23 \\
\hline 6 & Calcium & 20.26 & 4.48 & 75.27 & 16 & Plant height & 27.30 & 33.77 & 38.93 \\
\hline 7 & Magnesium & 57.47 & 9.63 & 32.90 & 17 & No. of branches & 27.74 & 4.04 & 68.23 \\
\hline 8 & Fruit length & 20.64 & 18.76 & 60.61 & 18 & No. of clusters & 31.10 & 4.82 & 64.09 \\
\hline 9 & Fruit diameter & 35.04 & 6.45 & 58.51 & 19 & $\begin{array}{l}\text { No.of } \\
\text { fruits/cluster }\end{array}$ & 36.34 & 9.54 & 54.12 \\
\hline 10 & Fruit weight & 24.44 & 2.24 & 73.32 & 20 & Yield/plant & 25.00 & 19.30 & 55.71 \\
\hline
\end{tabular}

Table Vl: Variance due to general and specific combining ability interaction towards variation in tomato hybrids 


\begin{tabular}{|c|c|c|c|c|c|c|c|c|c|}
\hline SI. No. & Characters & $\sigma^{2} \mathrm{GCA}$ & $\sigma^{2} S C A$ & $\sigma^{2} \mathrm{GCA} / \sigma^{2} \mathrm{SCA}$ & SI. No. & Characters & $\sigma^{2} \mathrm{GCA}$ & $\sigma^{2} S C A$ & $\sigma^{2} \mathrm{GCA} \sigma^{2} \mathrm{SCA}$ \\
\hline 1 & TSS & 0.02 & 0.04 & 0.5 & 11 & Fruit firmness & $0.03^{*}$ & 0.01 & 3.00 \\
\hline 2 & $\mathrm{pH}$ & 0.01 & $0.01 *$ & 1.00 & 12 & Pericarp thickness & 0.06 & 0.16 & 0.38 \\
\hline 3 & Lycopene & 0.53 & $1.72^{\star \star}$ & 0.31 & 13 & Pulp content & $7.66^{* \star}$ & 10.57 & 0.72 \\
\hline 4 & Ascorbic acid & $8.18^{*}$ & $4.54^{\star \star}$ & 1.80 & 14 & Locule number & 0.04 & 0.10 & 0.40 \\
\hline 5 & Titratable acidity & 0.01 & $0.02^{*}$ & 0.50 & 15 & Shelf life & 2.51 & 6.48 & 0.39 \\
\hline 6 & Calcium & 2.06 & $18.83^{\star \star}$ & 0.11 & 16 & Plant height & 0.01 & $0.01 *$ & 1.00 \\
\hline 7 & Magnesium & 8.86 & $13.03^{\star \star}$ & 0.38 & 17 & No. of branches & 0.03 & 0.56 & 0.05 \\
\hline 8 & Fruit length & 5.08 & $24.61^{\star \star}$ & 0.20 & 18 & No. of clusters & 0.43 & $2.33^{\star \star}$ & 0.18 \\
\hline 9 & Fruit diameter & 0.70 & 3.74 & 0.19 & 19 & No. of fruits/cluster & 0.20 & $0.72^{\star \star}$ & 0.28 \\
\hline 10 & Fruit weight & 12.45 & $113.49 * *$ & 0.11 & 20 & Yield/plant & 34646 & $1355536.83^{\star *}$ & 0.06 \\
\hline
\end{tabular}

\subsection{General combining ability effects and overall general combining ability status}

Line IIHR 2358 was the best with the highest GCA effects in a positive direction for lycopene, ascorbic acid, number of fruits/cluster (Table VII). Apart from this, it was a good general combiner for $\mathrm{pH}$, fruit weight, shelf life, plant height, and yield/plant in a positive direction. The lines IIHR 2349 had significant GCA effects in the desired direction for fruit length, weight, and firmness. Line IIHR 2357 was a very good source of favorable genes for TSS, fruit length, and shelf life. The entire testers were good general combiners for lycopene, titratable acidity, calcium, and magnesium. Arka Saurabh had high general combining ability effects for $\mathrm{pH}$, fruit length, plant height, number of clusters, and number of fruits/cluster. Best lines and testers for each character with significant GCA effects in the desired direction are presented in Table VIII. In the GCA effects of the parents, the line IIHR 2357 and the tester Arka Vikas had high $(\mathrm{H})$ overall GCA status (Table IX).

\subsection{Specific combining ability effects and overall specific combining ability effects of crosses}

The hybrids from different combinations of the parents with high or low GCA effects are referred to as $H \times H$ (high $\times$ high), $H \times L$ (high $\times$ low), and $L \times L$ (low $\times$ low) combinations (Table X). Among nine hybrids, IIHR $2349 \times$ Arka Vikas $(\mathrm{L} \times \mathrm{H})$ was found good specific combiner for number of clusters, and yield/plant (Table XI). The hybrid, IIHR $2349 \times$ Arka Ahuti $(L \times L)$ was a good specific combiner for calcium, magnesium, fruit length, and weight in a desirable direction. For lycopene, ascorbic acid, calcium, and magnesium, the hybrid IIHR $2349 \times$ Arka Saurabh $(L \times L)$ was found superior in a desirable direction. Concerning titratable acidity, the hybrid IIHR $2357 \times$ Arka Ahuti $(\mathrm{H} \times \mathrm{L})$, was the best specific combiner as it exhibited high positive significant SCA effects. As far as ascorbic acid, fruit length, and number of fruits/cluster concerned, the hybrid IIHR $2357 \times$ Arka Saurabh (H $\times \mathrm{L}$ ) was the top specific combiners with SCA effects in a desirable direction.

Out of nine crosses, four crosses had high overall specific combining ability status. The crosses IIHR $2349 \times$ Arka Vikas (H $\times$ L), IIHR $2349 \times$ Arka Saurabh (L $\times L$ ) were the best overall specific combiner followed by IIHR $2358 \times$ Arka Ahuti $(L \times L)$ and IIHR $2357 \times$ Arka Ahuti $(H \times L)($ Table XII). Based on SCA effects, crosses were classified into $\mathrm{H} \times \mathrm{H}$ (both the parents with high overall GCA status), $\mathrm{H} \times \mathrm{L}$ (one parent with high and other with low overall GCA status), and $\mathrm{L} \times$ $\mathrm{L}$ (both the parents with low overall GCA status). Out of nine crosses, one cross was $\mathrm{H} \times \mathrm{H}$ (IIHR $2357 \times$ Arka Vikas) involved both the parents with good overall general combining ability. Four crosses were $H \times L$ or $L \times H$ and the remaining four crosses were $L \times L$.

Table VII: Estimates of GCA effects of lines and testers for fruit biochemical, morpho-physiological and yield traits 


\begin{tabular}{|c|c|c|c|c|c|c|c|c|c|c|c|}
\hline $\begin{array}{l}\text { SI. } \\
\text { No. }\end{array}$ & $\begin{array}{l}\text { Lines and } \\
\text { Testers }\end{array}$ & TSS & $\mathrm{pH}$ & Lycopene & $\begin{array}{l}\text { Ascorbic } \\
\text { acid }\end{array}$ & $\begin{array}{l}\text { Titratable } \\
\text { acidity }\end{array}$ & Calcium & Magnesium & $\begin{array}{l}\text { Fruit } \\
\text { length }\end{array}$ & $\begin{array}{l}\text { Fruit } \\
\text { diameter }\end{array}$ & $\begin{array}{l}\text { Fruit } \\
\text { weight }\end{array}$ \\
\hline 1 & IIHR 2349 & -0.14 & 0.03 & $0.34 * \star$ & $2.34 \star \star$ & -0.02 & 1.06 ** & $3.46^{\star *}$ & $2.48^{*}$ & -0.82 & $3.91 *$ \\
\hline 2 & IIHR 2358 & -0.07 & $0.07 *$ & 0.80 ** & $4.48^{\star \star}$ & 0.02 & 1.06 ** & $0.75^{\star \star}$ & 0.03 & 1.73 & $5.84^{\star \star}$ \\
\hline 3 & IIHR 2357 & $0.21^{*}$ & 0.04 & $0.47^{\star *}$ & 2.14 ** & -0.01 & $2.12^{\star \star}$ & $4.22^{\star \star}$ & $2.45^{\star}$ & -0.91 & -1.94 \\
\hline 4 & $\begin{array}{l}\text { Arka } \\
\text { Vikas }\end{array}$ & 0.16 & 0.04 & 0.41 ** & $1.17 \star \star$ & $0.11 *$ & 0.91 ** & $1.65^{\star \star}$ & -0.23 & 0.48 & 1.79 \\
\hline 5 & $\begin{array}{l}\text { Arka } \\
\text { Ahuti }\end{array}$ & -0.04 & 0.04 & $0.86^{\star \star}$ & $1.13^{\star *}$ & $0.09 *$ & $0.80 * *$ & $1.53^{\star \star}$ & $2.45^{\star}$ & -0.73 & -1.04 \\
\hline 6 & $\begin{array}{l}\text { Arka } \\
\text { Saurabh }\end{array}$ & -0.12 & $0.08^{*}$ & $0.46^{\star \star}$ & -0.03 & $0.20 \star \star$ & $0.11^{\star \star}$ & $0.13^{\star \star}$ & $2.23^{*}$ & 0.26 & -0.76 \\
\hline \multicolumn{2}{|c|}{ S. E. \pm} & 0.08 & 0.03 & 0.04 & 0.14 & 0.03 & 0.01 & 0.02 & 0.85 & 0.79 & 1.43 \\
\hline \multicolumn{2}{|c|}{ C.D. $5 \%$} & 0.2 & 0.06 & 0.09 & 0.32 & 0.08 & 0.01 & 0.05 & 1.95 & 1.82 & 3.31 \\
\hline \multicolumn{2}{|c|}{ C.D. $1 \%$} & 0.28 & 0.09 & 0.14 & 0.47 & 0.11 & 0.01 & 0.08 & 2.84 & 2.65 & 4.81 \\
\hline $\begin{array}{l}\text { SI. } \\
\text { No. }\end{array}$ & $\begin{array}{l}\text { Lines and } \\
\text { Testers }\end{array}$ & $\begin{array}{l}\text { Fruit } \\
\text { firmness }\end{array}$ & $\begin{array}{l}\text { Pericarp } \\
\text { thickness }\end{array}$ & $\begin{array}{l}\text { Pulp } \\
\text { content }\end{array}$ & $\begin{array}{l}\text { Locule } \\
\text { number }\end{array}$ & Shelf life & $\begin{array}{l}\text { Plant } \\
\text { height }\end{array}$ & $\begin{array}{l}\text { No. of } \\
\text { branches }\end{array}$ & $\begin{array}{l}\text { No. of } \\
\text { clusters }\end{array}$ & $\begin{array}{l}\text { No. of } \\
\text { fruits/cluster }\end{array}$ & Yield/plant \\
\hline 1 & IIHR 2349 & $0.33^{*}$ & 0.31 & 2.57 & 0.02 & 0.3 & 0.03 & 0.07 & $0.98^{\star \star *}$ & $0.49 \star \star$ & 43.96 \\
\hline 2 & IIHR 2358 & -0.08 & 0.01 & -4.37 & -0.03 & 2.60 * & $0.07 *$ & -0.51 & $0.63^{\star \star}$ & $0.62^{\star \star}$ & 233.41 * \\
\hline 3 & IIHR 2357 & -0.25 & -0.33 & 1.8 & 0.02 & $2.30 *$ & 0.04 & 0.44 & $0.14^{\star \star}$ & $0.14^{\star *}$ & $189.46^{*}$ \\
\hline 4 & $\begin{array}{l}\text { Arka } \\
\text { Vikas }\end{array}$ & -0.04 & 0.38 & -3.33 & 0.02 & 0.3 & 0.05 & 0.11 & $0.36^{\star \star}$ & 0.03 & $217.22^{*}$ \\
\hline 5 & $\begin{array}{l}\text { Arka } \\
\text { Ahuti }\end{array}$ & 0.09 & -0.3 & 2.5 & 0.12 & -0.86 & 0.04 & 0.11 & -0.03 & 0.31 ** & 124.04 \\
\hline 6 & $\begin{array}{l}\text { Arka } \\
\text { Saurabh }\end{array}$ & -0.04 & -0.08 & 0.83 & -0.13 & 0.56 & $0.08^{*}$ & -0.21 & $0.33^{\star \star}$ & $0.28 * \star$ & 93.18 \\
\hline \multicolumn{2}{|c|}{ S. E. \pm} & 0.11 & 0.23 & 2.02 & 0.21 & 0.9 & 0.03 & 0.31 & 0.06 & 0.04 & 79.02 \\
\hline \multicolumn{2}{|c|}{ C.D. $5 \%$} & 0.26 & 0.53 & 4.65 & 0.48 & 2.09 & 0.06 & 0.72 & 0.14 & 0.09 & 182.23 \\
\hline \multicolumn{2}{|c|}{ C.D. $1 \%$} & 0.38 & 0.76 & 6.77 & 0.7 & 3.03 & 0.09 & 1.05 & 0.2 & 0.14 & 265.15 \\
\hline
\end{tabular}

Where, $*$ Significant at $p=0.05, * \star$ Significant at $p=0.01$, SE: Standard error, C.D.: Critical difference

Table VIII: Best tomato lines and testers with significant GCA effects in the desirable direction

\begin{tabular}{|c|c|c|c|c|c|c|c|}
\hline $\begin{array}{l}\text { Sl. } \\
\text { No. }\end{array}$ & Characters & Lines & Testers & $\begin{array}{l}\text { Sl. } \\
\text { No. }\end{array}$ & Characters & Lines & Testers \\
\hline 1 & TSS & IIHR $2357\left(0.21^{\star}\right)$ & Arka Vikas (0.16) & 11 & Fruit firmness & IIHR $2349\left(0.33^{\star}\right)$ & Arka Ahuti (0.09) \\
\hline 2 & $\mathrm{pH}$ & IIHR 2358 (0.07*) & Arka Saurabh $\left(0.08^{\star}\right)$ & 12 & Pericarp thickness & IIHR 2349 (0.31) & Arka Vikas (0.39) \\
\hline 3 & Lycopene & $\begin{array}{l}\text { IIHR } 2358 \\
\left(0.80^{\star *}\right)\end{array}$ & Arka Ahuti $\left(0.86^{\star \star}\right)$ & 13 & Pulp content & IIHR 2349 (2.57) & Arka Ahuti (2.50) \\
\hline 4 & Ascorbic acid & $\begin{array}{l}\text { IIHR } 2358 \\
\left(4.48^{\star *}\right)\end{array}$ & Arka Vikas $\left(1.17^{\star \star}\right)$ & 14 & Locule number & IIHR $2358(-0.03)$ & Arka Saurabh (-0.13) \\
\hline 5 & $\begin{array}{l}\text { Titratable } \\
\text { acidity }\end{array}$ & IIHR 2358 (0.02) & $\begin{array}{l}\text { Arka Saurabh } \\
\left(0.11^{\star \star}\right)\end{array}$ & 15 & Shelf life & IIHR 2358 (2.60*) & Arka Saurabh (0.57) \\
\hline 6 & Calcium & $\begin{array}{l}\text { IIHR } 2357 \\
\left(2.12^{\star *}\right)\end{array}$ & Arka Vikas $\left(0.91^{\star \star}\right)$ & 16 & Plant height & IIHR $2358\left(0.07^{\star}\right)$ & $\begin{array}{l}\text { Arka Saurabh } \\
\left(0.08^{\star}\right)\end{array}$ \\
\hline 7 & Magnesium & $\begin{array}{l}\text { IIHR } 2357 \\
\left(4.22^{\star \star}\right)\end{array}$ & Arka Vikas $\left(1.65^{\star \star}\right)$ & 17 & No. of branches & IIHR 2357 (0.44) & Arka Vikas (0.11) \\
\hline 8 & Fruit length & IIHR 2349 (2.48*) & Arka Ahuti $\left(2.46^{\star}\right)$ & 18 & No. of clusters & IIHR $2349\left(0.98^{\star \star}\right)$ & Arka Vikas $\left(0.36^{\star \star}\right)$ \\
\hline 9 & Fruit diameter & IIHR 2358 (1.73) & Arka Vikas (0.48) & 19 & $\begin{array}{l}\text { No. of } \\
\text { fruits/cluster }\end{array}$ & IIHR $2358\left(0.63^{\star \star}\right)$ & Arka Ahuti $\left(0.31^{\star \star}\right)$ \\
\hline 10 & Fruit weight & $\begin{array}{l}\text { IIHR } 2358 \\
\left(5.84^{\star *}\right)\end{array}$ & Arka Vikas (1.79) & 20 & Yield/plant & $\begin{array}{l}\text { IIHR } 2358 \\
(233.41 *)\end{array}$ & Arka Vikas $\left(217.22^{*}\right)$ \\
\hline
\end{tabular}


Table IX: Overall general combining ability status of tomato lines and testers

\begin{tabular}{|llllllll|}
\hline SI. No. & Lines & Rank & GCA Status & SI. No. & Testers & Rank & GCA Status \\
\hline 1 & IIHR 2349 & 44 & L & 1 & Arka Vikas & 32 & H \\
\hline 2 & IIHR 2358 & 43 & L & 2 & Arka Ahuti & 42 & L \\
\hline 3 & IIHR 2357 & 33 & H & 3 & Arka Saurabh & 46 & L \\
\hline Final Norm = 40 & & & Final Norm $=40$ & & \\
\hline
\end{tabular}

Table X: Estimates of SCA effects of crosses for fruit biochemical, morpho-physiological and yield traits 


\begin{tabular}{|c|c|c|c|c|c|c|c|c|c|c|c|}
\hline $\begin{array}{l}\text { Sl. } \\
\text { No. }\end{array}$ & Hybrids & TSS & $\mathrm{pH}$ & Lycopene & $\begin{array}{l}\text { Ascorbic } \\
\text { acid }\end{array}$ & $\begin{array}{l}\text { Titratable } \\
\text { acidity }\end{array}$ & Calcium & Magnesium & $\begin{array}{l}\text { Fruit } \\
\text { length }\end{array}$ & $\begin{array}{l}\text { Fruit } \\
\text { diameter }\end{array}$ & $\begin{array}{l}\text { Fruit } \\
\text { weight }\end{array}$ \\
\hline 1 & $\begin{array}{l}\text { IIHR } 2349 \text { x } \\
\text { Arka Vikas }\end{array}$ & -0.01 & -0.05 & $1.16^{* \star}$ & $1.15^{\star \star}$ & 0.05 & $0.68^{* *}$ & $0.65^{\star \star}$ & 1.53 & 0.83 & $05.87 *$ \\
\hline 2 & $\begin{array}{l}\text { IIHR } 2349 \text { x } \\
\text { Arka Ahuti }\end{array}$ & -0.08 & 0.03 & -0.11 & $1.23^{\star \star}$ & -0.08 & $4.89 \star \star$ & $3.53^{\star *}$ & $5.42 * \star$ & -1.61 & $12.48^{\star \star}$ \\
\hline 3 & $\begin{array}{l}\text { IIHR } 2349 \text { x } \\
\text { Arka } \\
\text { Saurabh }\end{array}$ & 0.09 & 0.02 & $1.27 * \star$ & $2.37^{\star \star}$ & 0.03 & $4.20 * \star$ & $4.17^{\star \star}$ & $3.89 *$ & 0.79 & $06.61^{*}$ \\
\hline 4 & $\begin{array}{l}\text { IIHR } 2358 \text { x } \\
\text { Arka Vikas }\end{array}$ & -0.16 & 0.04 & $0.17 *$ & -0.12 & 0.06 & $1.47^{\star \star}$ & $1.81^{\star \star}$ & -2.89 & -2.7 & 09.19 ** \\
\hline 5 & $\begin{array}{l}\text { IIHR } 2358 \text { x } \\
\text { Arka Ahuti }\end{array}$ & -0.08 & 0.06 & $0.96^{* *}$ & 0.25 & -0.11 & 2.31 ** & $1.20 * *$ & 1.36 & 2.43 & $07.76^{*}$ \\
\hline 6 & $\begin{array}{l}\text { IIHR } 2358 \text { x } \\
\text { Arka } \\
\text { Saurabh }\end{array}$ & 0.24 & -0.1 & $1.13^{\text {** }}$ & -0.13 & 0.05 & $3.78^{\star *}$ & 3.01 ** & 1.53 & 0.26 & 1.44 \\
\hline 7 & $\begin{array}{l}\text { IIHR } 2357 \text { x } \\
\text { Arka Vikas }\end{array}$ & 0.17 & 0.01 & 0.98 ** & $1.02 \star \star$ & -0.11 & $2.15^{\star \star}$ & $1.17 \star \star$ & 1.36 & 1.87 & 3.32 \\
\hline 8 & $\begin{array}{l}\text { IIHR } 2357 \text { x } \\
\text { Arka Ahuti }\end{array}$ & 0.16 & -0.1 & $0.85^{\star *}$ & $1.48^{\star *}$ & $0.19 *$ & $2.57^{\star \star}$ & $2.32 * *$ & $4.06^{*}$ & -0.82 & 4.72 \\
\hline 9 & $\begin{array}{l}\text { IIHR } 2357 \text { x } \\
\text { Arka } \\
\text { Saurabh }\end{array}$ & -0.33 & 0.08 & -0.14 & $2.50 \star \star$ & -0.08 & $0.42^{\star \star}$ & 1.16 ** & $5.42^{\star \star}$ & -1.05 & $08.04^{*}$ \\
\hline \multicolumn{2}{|c|}{ S. E. \pm} & 0.15 & 0.05 & 0.07 & 0.24 & 0.06 & 0.01 & 0.04 & 1.46 & 1.37 & 2.48 \\
\hline \multicolumn{2}{|c|}{ C.D. $5 \%$} & 0.34 & 0.11 & 0.16 & 0.56 & 0.13 & 0.01 & 0.09 & 3.38 & 3.15 & 5.73 \\
\hline \multicolumn{2}{|c|}{ C.D. $1 \%$} & 0.5 & 0.16 & 0.24 & 0.81 & 0.2 & 0.01 & 0.13 & 4.91 & 4.59 & 8.33 \\
\hline $\begin{array}{l}\text { SI. } \\
\text { No. }\end{array}$ & Hybrids & $\begin{array}{l}\text { Fruit } \\
\text { firmness }\end{array}$ & $\begin{array}{l}\text { Pericarp } \\
\text { thickness }\end{array}$ & $\begin{array}{l}\text { Pulp } \\
\text { content }\end{array}$ & $\begin{array}{l}\text { Locule } \\
\text { number }\end{array}$ & Shelf life & $\begin{array}{l}\text { Plant } \\
\text { height }\end{array}$ & $\begin{array}{l}\text { No. of } \\
\text { branches }\end{array}$ & $\begin{array}{l}\text { No. of } \\
\text { clusters }\end{array}$ & $\begin{array}{l}\text { No. of } \\
\text { fruits/cluster }\end{array}$ & Yield/plant \\
\hline 1 & $\begin{array}{l}\text { IIHR } 2349 \text { x } \\
\text { Arka Vikas }\end{array}$ & 0.03 & 0.21 & -1.21 & -0.05 & -0.33 & -0.05 & -0.51 & $1.73^{\star *}$ & 0.09 & 504.20 ** \\
\hline 2 & $\begin{array}{l}\text { IIHR } 2349 \text { x } \\
\text { Arka Ahuti }\end{array}$ & -0.11 & -0.52 & -0.09 & -0.05 & -2.03 & 0.03 & 1.08 & $1.29 \star \star$ & -0.03 & -143.89 \\
\hline 3 & $\begin{array}{l}\text { IIHR } 2349 \text { x } \\
\text { Arka } \\
\text { Saurabh }\end{array}$ & 0.09 & 0.31 & 1.3 & 0.1 & 2.37 & 0.02 & -0.57 & $0.44^{\star *}$ & -0.06 & 360.31 * \\
\hline 4 & $\begin{array}{l}\text { IIHR } 2358 \text { x } \\
\text { Arka Vikas }\end{array}$ & -0.15 & -0.03 & 1.01 & -0.05 & -2.37 & 0.04 & 0.29 & -0.14 & $0.62^{\star \star}$ & -306.04 \\
\hline 5 & $\begin{array}{l}\text { IIHR } 2358 \text { x } \\
\text { Arka Ahuti }\end{array}$ & 0.02 & 0.62 & -0.74 & -0.1 & 1.63 & 0.06 & -0.12 & $0.64^{* *}$ & $0.31^{\star *}$ & 116.58 \\
\hline 6 & $\begin{array}{l}\text { IIHR } 2358 \text { x } \\
\text { Arka } \\
\text { Saurabh }\end{array}$ & 0.13 & -0.6 & -0.26 & 0.15 & 0.73 & -0.1 & -0.17 & 0.51 ** & $0.93^{\star \star}$ & 189.46 \\
\hline 7 & $\begin{array}{l}\text { IIHR } 2357 \text { x } \\
\text { Arka Vikas }\end{array}$ & 0.13 & -0.19 & 0.21 & 0.1 & 2.7 & 0.01 & 0.21 & $1.59 * *$ & $0.71^{\star *}$ & -198.16 \\
\hline 8 & $\begin{array}{l}\text { IIHR } 2357 \text { x } \\
\text { Arka Ahuti }\end{array}$ & 0.09 & -0.1 & 0.83 & 0.15 & 0.4 & -0.1 & -0.96 & $0.64^{* *}$ & $0.28^{\star \star}$ & 27.31 \\
\hline 9 & $\begin{array}{l}\text { IIHR } 2357 \text { x } \\
\text { Arka } \\
\text { Saurabh }\end{array}$ & -0.22 & 0.28 & -1.04 & -0.25 & -3.1 & 0.08 & 0.74 & $0.94^{\star *}$ & $0.99 * *$ & 170.85 \\
\hline \multicolumn{2}{|c|}{ S. E. \pm} & 0.2 & 0.4 & 3.5 & 0.36 & 1.57 & 0.05 & 0.54 & 0.1 & 0.07 & 136.87 \\
\hline \multicolumn{2}{|c|}{ C.D. $5 \%$} & 0.45 & 0.91 & 8.06 & 0.84 & 3.61 & 0.11 & 1.24 & 0.24 & 0.16 & 315.62 \\
\hline \multicolumn{2}{|c|}{ C.D. $1 \%$} & 0.66 & 1.32 & 11.73 & 1.22 & 5.26 & 0.16 & 1.81 & 0.34 & 0.24 & 459.25 \\
\hline
\end{tabular}

Table XI: Best tomato crosses with significant SCA effects in the desirable direction 


\begin{tabular}{|c|c|c|c|c|c|}
\hline SI. No. & Characters & Crosses & SI. No. & Characters & Crosses \\
\hline \multirow[t]{2}{*}{1} & \multirow[t]{2}{*}{ TSS } & \multirow[t]{2}{*}{ IIHR 2358 x Arka Saurabh (0.24) } & \multirow[t]{2}{*}{11} & \multirow[t]{2}{*}{ Fruit firmness } & IIHR 2358 x Arka Saurabh (0.13) \\
\hline & & & & & IIHR 2357 x Arka Vikas (0.13) \\
\hline 2 & $\mathrm{pH}$ & IIHR 2357 x Arka Saurabh (0.08) & 12 & Pericarp thickness & IIHR 2358 x Arka Ahuti (0.62) \\
\hline \multirow[t]{2}{*}{3} & \multirow[t]{2}{*}{ Lycopene } & IIHR 2349 x Arka Saurabh (1.27**) & \multirow[t]{2}{*}{13} & \multirow[t]{2}{*}{ Pulp content } & \multirow[t]{2}{*}{ IIHR 2349 x Arka Saurabh (1.30) } \\
\hline & & IIHR 2349 x Arka Vikas (1.16**) & & & \\
\hline \multirow[t]{2}{*}{4} & \multirow[t]{2}{*}{ Ascorbic acid } & IIHR 2357 x Arka Saurabh (2.50**) & \multirow[t]{2}{*}{14} & \multirow[t]{2}{*}{ Locule number } & \multirow[t]{2}{*}{ IIHR 2357 x Arka Saurabh (-0.25) } \\
\hline & & IIHR 2349 x Arka Saurabh (2.37**) & & & \\
\hline \multirow[t]{2}{*}{5} & \multirow[t]{2}{*}{ Titratable acidity } & \multirow[t]{2}{*}{ IIHR 2357 x Arka Ahuti $(0.19 *)$} & \multirow[t]{2}{*}{15} & \multirow[t]{2}{*}{ Shelf life } & IIHR 2357 x Arka Vikas (2.70) \\
\hline & & & & & IIHR 2349 x Arka Saurabh (2.37) \\
\hline \multirow[t]{2}{*}{6} & \multirow[t]{2}{*}{ Calcium } & IIHR 2349 x Arka Ahuti (4.89**) & \multirow[t]{2}{*}{16} & \multirow[t]{2}{*}{ Plant height } & \multirow[t]{2}{*}{ IIHR 2357 x Arka Saurabh (0.08) } \\
\hline & & IIHR 2349 x Arka Saurabh (4.20**) & & & \\
\hline \multirow[t]{2}{*}{7} & \multirow[t]{2}{*}{ Magnesium } & IIHR 2349 x Arka Saurabh (4.17**) & \multirow[t]{2}{*}{17} & \multirow[t]{2}{*}{ No. of branches } & \multirow[t]{2}{*}{ IIHR 2349 x Arka Ahuti (1.08) } \\
\hline & & IIHR 2349 x Arka Ahuti (3.53**) & & & \\
\hline \multirow[t]{2}{*}{8} & \multirow[t]{2}{*}{ Fruit length } & IIHR 2349 x Arka Ahuti (5.42**) & \multirow[t]{2}{*}{18} & \multirow[t]{2}{*}{ No. of clusters } & IIHR 2349 x Arka Vikas (1.73**) \\
\hline & & IIHR 2357 x Arka Saurabh (5.42**) & & & IIHR 2357 x Arka Vikas (1.59**) \\
\hline \multirow[t]{2}{*}{9} & \multirow[t]{2}{*}{ Fruit diameter } & IIHR 2358 x Arka Ahuti (2.43) & 19 & No. of fruits/cluster & IIHR 2357 x Arka Saurabh (0.99**) \\
\hline & & & & & IIHR 2358 x Arka Saurabh $\left(0.93^{\star \star}\right)$ \\
\hline 10 & Fruit weight & IIHR 2349 x Arka Ahuti $\left(12.48^{\star \star}\right)$ & 20 & Yield/plant & 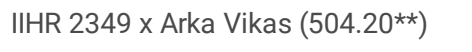 \\
\hline & & & & & IIHR 2349 x Arka Saurabh (360.31**) \\
\hline
\end{tabular}

Table XII: Overall specific combining ability status of tomato crosse

\begin{tabular}{|lllllllll|}
\hline Lines & \multicolumn{3}{l}{ Tester } & & & \\
& \cline { 3 - 8 } & \multicolumn{2}{l}{ Arka Vikas (H) } & \multicolumn{2}{ll}{ Arka Ahuti (L) } & Arka Saurabh (L) \\
\cline { 3 - 8 } & & Rank & SCA status & Rank & SCA status & Rank & SCA status \\
\hline IIHR 2349 & (L) & 79 & H & 116 & L & 79 & H \\
IIHR 2358 & (L) & 104 & L & 80 & H & 111 & L \\
\hline IIHR 2357 & (H) & 105 & L & 97 & H & 106 & L \\
\hline Final Norm & $=97.44$ & & & & & \\
\hline
\end{tabular}

Where,

$\mathrm{H}=$ High overall SCA status $(\mathrm{H})=$ High overall GCA status
$\mathrm{L}=$ Low overall SCA status $(\mathrm{L})=$ Low overall GCA status

\section{Discussion}

The substantial variability between the lines and testers indicated the contrasting nature of lines and testers for fruit shelf life and yield/plant. The line IIHR 2349, the tester Arka Vikas and the hybrid IIHR $2349 \times$ Arka Saurabh were superior for most studied traits. Significant differences observed among the genotypes for most studied traits indicated the presence of a considerable amount of genetic variability and justified the selection of parents for the study. Significant differences observed among the lines for $\mathrm{pH}$, lycopene, ascorbic acid, calcium, magnesium, fruit length, locule number, plant height, number of cluster, and number of fruit/cluster indicated the significance of additive variance for these traits. Significance of parents v/s hybrids revealed the heterosis and significance of hybrids indicated the diverse performance of different cross combinations. 
The significance of mean sum of squares due to lines indicated the significance of additive variance for ascorbic acid, fruit firmness, and pulp content. The significance of Line $\times$ Tester interaction variance indicated the significance of dominance variance and the presence of heterosis for lycopene, ascorbic acid, titratable acidity, calcium, magnesium, fruit length, diameter, weight, number of branches, number of clusters, and yield/plant. The combining ability of lines differed based on the tester involved in the cross combination which was amply reflected by significant differences for crosses.

The lines contributed to the bulk of the variation observed in hybrids which were higher for TSS, ascorbic acid, calcium, magnesium, fruit length, diameter, weight, firmness, pulp content, shelf life, number of branches, number of clusters, number of fruits and yield/plant. Lines have an important role in the variation among the hybrids, while selecting the germplasm lines, emphasis should be given to the lines which are used as female parents. However, the same thing cannot be done concerning line $\times$ tester interaction as it is not under the control of the breeder, but it depends on the specific manner in which the lines and testers interact. Therefore, most care should be taken while selecting the lines for hybridization.

The predominance of non-additive gene action exhibited by lycopene, titratable acidity, pericarp thickness (Dhatt et al. 2003; Joshi et al. 2005), yield/plant (Bhutani and Kalloo 1983; Das et al. 2020; Pavan and Gangaprasad 2022), TSS (Kalloo et al. 1974; Bhatt et al. 2001), calcium, magnesium, fruit length, diameter, weight, pulp content, locule number (Dhatt et al. 2003), shelf life (Roopa et al. 2001; Dhatt et al. 2003; Garg et al. 2008; Pavan and Gangaprasad 2022), number of branches, number of clusters, number of fruit/cluster (Pavan and Gangaprasad, 2022) which can be exploited through hybrid development programs as the GCA/SCA ratio was less than one. Contrasting findings of additive gene action were cited for locule number, shelf life (Rodriguez et al., 2010), yield/plant (Katoch and Vidyasagar 2004), number of fruit/cluster (Mondal et al. 2009), and lycopene (Suo et al. 2010). The additive and non-additive gene effects for fruit quality and yield traits were reported by Gaikwad and Cheema (2009) and Akhtar and Hazra (2013). pH, ascorbic acid, fruit firmness, and plant height were governed by additive gene action which can be exploited through varietal development programs as the GCA/SCA ratio was more than one. The contrasting results of dominant gene action were reported for pH and plant height (Das et al. 2020; Pavan and Gangaprasad 2022), ascorbic acid, and fruit firmness (Bhatt et al. 2001; Pavan and Gangaprasad 2022).

The per se performance of a parent is not always a true indicator of its potentiality in a hybrid combination. Therefore, general combining ability, which is the breeding value of the parent expressed as a deviation from the population mean has proved as a useful tool for choosing the potential parents for hybrid development (Singh and Asati 2011). Among the parents with significant GCA effects, the one with a higher magnitude of GCA effects is considered superior to those with a lower magnitude (Technow 2019; Yu et al. 2020). An overall appraisal of GCA effects revealed that all the lines were good general combiners for lycopene, ascorbic acid, calcium, magnesium, number of clusters, and number of fruits/cluster. The Arka Saurabh had high general combining ability effects for $\mathrm{pH}$, fruit length, plant height, number of clusters, and number of fruits/cluster proved to be the best combiner in producing heterotic hybrids. The high overall GCA status of the line IIHR 2357 and the tester Arka Vikas revealed that $33.33 \%$ of lines and testers proved to be high overall good general combiners which intern suggested their ability to transmit additive genes in the desired direction for the majority of the traits under study. However, these lines and testers should be evaluated further to confirm their superiority. Similar results were reported by Bhatt et al. (2001), Dhatt et al. (2003), Kansouh and Zakher (2011), Yogendra and Gowda (2013).

The specific combining ability is used to designate those cases in which specific combinations do relatively better or worse than would be expected from the average performance of the lines involved. The SCA is controlled by dominance and nonallelic gene interactions. The high GCA effects of parents may produce hybrids with low SCA effects and vice versa. Therefore, when selecting elite parents for crosses, understanding and accounting for the relationship between the GCA of the parents, the SCA of the crosses and the dominant type of combining ability are key to improving breeding efficiency based on combining ability (Liu et al. 2019). Both the crosses, IIHR $2349 \times$ Arka Ahuti $(L \times L)$ and IIHR $2349 \times$ Arka Saurabh $(L \times L)$ involved low combiners which showed the importance of overdominance and epistasis in the inheritance of lycopene, ascorbic acid, calcium, magnesium, fruit length, and weight. Involvement of high and low combiners in the crossing revealed the significance of non-additive gene action governing the traits. Hence, hybrid development will be effective. The findings of Singh and Asati (2011), Yadav et al. (2013), and Adhi Shankar et al. (2014) would substantially support the present results.

Out of nine crosses, four crosses had high overall specific combining ability status indicated that $45.55 \%$ of hybrids were assigned high $(\mathrm{H})$ overall specific combiners. The cross IIHR $2357 \times$ Arka Vikas, involved both the parents with good overall general combining ability $(\mathrm{H} \times \mathrm{H})$. Therefore additive gene action may be imperative and simple selection in segregating generation will be effective and the reliance should be placed on mass selection and progeny selection. Four crosses were $\mathrm{H} \times \mathrm{L}$ or $\mathrm{L} \times \mathrm{H}$ indicated the presence of non-additive gene action which is a prerequisite for launching a heterosis breeding. The remaining four crosses were $L \times L$ showed the significance of overdominance and epistatic gene action in the inheritance. Hence, intensive selection for SCA in segregating generations will be effective in the genetic improvement of fruit quality traits governing shelf life.

The two promising hybrids were identified based on their per se performance for yield and shelf life. The hybrid IIHR $2349 \times$ Arka Vikas recorded a significantly higher yield potential of $2850.50 \mathrm{~g} /$ plant with significantly higher standard heterosis over standard check Arka Rakshak (52.27\%), and it had 24.70 days of shelf life. Higher heterosis for yield/plant in this hybrid may be attributed to higher mean performance for number of clusters/plant (11.05) which is further reiterated by significantly higher standard heterosis for the same trait (8.87\%). Another promising hybrid IIHR $2349 \times$ Arka Saurabh recorded a significantly higher shelf life of 29.40 days with significantly higher standard heterosis over standard check Arka Rakshak (26.45\%), and it had a yield potential of $2397.50 \mathrm{~g} /$ plant. Higher heterosis for shelf life in this hybrid may be contributed by significantly higher mean performance for fruit magnesium content $(17.79 \mathrm{mg} / 100 \mathrm{~g})$ and lycopene content $(4.59 \mathrm{mg} / 100 \mathrm{~g})$ which is further evident by significantly higher standard heterosis for magnesium content (154.14\%) and lycopene content (128.36\%). These promising hybrids need to be tested at preliminary yield trials and multilocation trials to assess their performance and stability before commercialization.

\section{Conclusions}


The crosses, IIHR $2349 \times$ Arka Vikas and IIHR $2349 \times$ Arka Saurabh were promising hybrids for high yield and shelf life. Significant differences among the genotypes indicated considerable genetic variability for fruit quality traits. The estimates of SCA variance was higher as compared to GCA variance. The non-additive gene action was exhibited for lycopene, titratable acidity, TSS, fruit length, diameter, weight, locule number, number of branches, number of clusters, number of fruit/cluster and yield/plant which can be exploited through hybrid development and additive gene action shown for $\mathrm{pH}$, ascorbic acid, fruit firmness, and plant height can be exploited through varietal development. The study underlined the significance of non-additive gene action for shelf life, pericarp thickness, calcium, magnesium, pulp content. The involvement of low combiners in hybridization signified over dominance and epistasis hence intensive selection for SCA in segregating generations will be effective. Involvement of high and low combiners in the crossing revealed the significance of non-additive gene action which is a prerequisite for heterosis breeding. The involvement of high combiners in crossing signified the importance of additive gene action and simple selection in segregating generation will be effective with more reliance on mass selection and progeny selection.

\section{Declarations}

\section{Acknowledgements}

We are grateful to Dr. Sadashiva A.T., Scientist, Division of Vegetable crops and Dr. Shivashankara K.S., Scientist, Division of Plant Physiology and Biochemistry, ICAR-IIHR, Bengaluru for providing Tomato seed materials, laboratory facility and Mr. Guruprasad, C.S., Food Corporation of India, K.R. Nagara, Mysuru for valuable guidance in research. We gratefully acknowledges the Indian Council of Agricultural Research (ICAR) financial support for pursuing Ph.D. degree.

\section{Funding}

The authors declare that no funds, grants, or other support were received during the preparation of this manuscript.

\section{Competing Interests}

The authors have no relevant financial or non-financial interests to disclose.

\section{Author Contributions}

All authors contributed to the study conception and design. Material preparation, data collection and analysis were performed by Pavan, Gangaprasad. The first draft of the manuscript was written by Pavan and all authors commented on previous versions of the manuscript. All authors read and approved the final manuscript.

\section{References}

Adhi shankar RV, Reddy SK, Sujatha M, Pratap, M (2014) Studies on combining ability and gene action studies for yield and yield contributing traits in tomato (Solanum lycopersicum L.). Helix 6: 431 - 435

Akhtar S, Hazra P (2013) Nature of gene action for fruit quality characters of tomato (Solanum lycopersicum). Afr J Biotech 12:2869-2875

Arab L, Steck S (2000) Lycopene and cardiovascular disease. American J Clinical Nutri 71 (6):1691S-1695S

Arah IK, Amaglo H, Kumah EK, Ofori H (2015) Preharvest and postharvest factors affecting the quality and shelf life of harvested tomatoes: a mini review. Int J Agron. https://doi.org/10.1155/2015/478041

Arunachalam V (1974) Fallacy behind the use of modified line x tester design. Indian J Genet 34: 280-287

Arunachalam V, Bandopadhyay A (1979) Are "Multilple cross Multiple pollen hybrids" an answer for productive populations in Brassica compestries variety brown serson? Theor Appl Genet 54: 203-207

Association of Official Analytical Chemists (2000) Official Methods of Analysis, $17^{\text {th }}$ edn, 942 . Titratable acidity of fruit products, p.15.

Association of Official Analytical Chemists (2006) Official Methods of Analysis, Ascorbic acid, 967.21, 45.1.14. AOAC International, Gaithersburg.

Bhatt RP, Biswas VR, Kumar N (2001) Heterosis, combining ability and genetics for vitamin C, total soluble solids and yield in tomato (Lycopersicon esculentum) at $1700 \mathrm{~m}$ altitude. J Agric Sci 137(1): 71-75

Bhutani RD, Kalloo G (1983) Genetics of carotenoids and lycopene in tomato (L. esculentum Mill.). Genetic Agrar 37: 1-6

Colombani Salliba, Cause M, Langlois D, Philouze J, Buret M (2001) Genetic analysis of organoleptic quality in fresh market tomato, Mapping QTLs for physical and chemical traits. Theor Appl Genet 102:259-272

Das, Ipsita, Hazra, Pranab, Longjam, Mrinalini, Bhattacharjee, Tridip, Kumar Maurya, Praveen, Banerjee, Swadesh, (2020) Genetic control of reproductive and fruit quality traits in crosses involving cultivars and induced mutants of tomato (Solanum lycopersicum L.). J Genet 99:56 
Delina T, Mahendran (2009) Physico-Chemical properties of mature green tomatoes with pectin during storage and ripening. Trop Agric Res Ext 12 (2):110112

Dhatt AS, Singh S, Dhaliwal MS (2003) Genetic analysis of shelf life and firmness of tomato using rin, nor and alc lines. J Genet Breed 57:313-318

Freeman BB, Reimers K (2011) Tomato consumption and health: emerging benefits. American J Lifestyle Medicine 5(2):182-191

Gaikwad AK, Cheema DS (2009) Heterosis for yield in heat tolerant tomato lines. Crop Improv 36 (1):55-59

Garg NS, Cheema DS, Dhatt AS (2007) Combining ability analysis involving rin, nor and alc alleles in tomato under late planting conditions. Adv Hort Sci 21(2):59-67

Garg NS, Cheema DS, Dhatt AS (2008) Genetics of yield, quality and shelf life characteristics in tomato under normal and late planting conditions. Euphytica 159:275-288

Hayes HK, Immer FR, Smith DC (1955) Methods of Plant Breeding. Mc. Graw Hill Book Co., Inc., New York. 551pp

Joshi A, Thakur MC, Kohli UK (2005) Heterosis and combining ability for shelf life, whole fruit firmness and related traits in tomato. Indian J Hortic 62(1): 3336

Kalloo G, Singh RK, Bhutani RD (1974) Combining ability studies in tomato (L. esculentum Mill.). Theor Appl Genet $44: 358$ - 63

Kansouh AM, Zakher AG (2011) Gene action and combining ability in tomato (Lycopersicon esculentum Mill.) by line x tester analysis. J Plant Production Mansoura Univ 2(2): 213-227

Katoch V, Vidyasagar (2004) Genetic studies on yield and its components in tomato. J Appl Hort 6:45-47

Kempthorne (1957) An introduction to genetic statistics. John Wiley and Sons. Inc., New York, 458-471pp

Lichtenthaler HK (1987) Chlorophylls and carotenoids: pigments of photosynthetic biomembranes. Meth Enzymol 148:350-382

Lismaíra Gonçalves Caixeta Garcia, Edson Pablo da Silva, Carlos de Melo Silva Neto, Eduardo Valério de Barros Vilas Boas, Eduardo Ramirez Asuieri, Clarissa Damiani, Flávio Alves da Silva (2019) Effect of the addition of calcium chloride and different storage temperatures on the post-harvest of jabuticaba variety Pingo de Mel. Food Sci Tech 39(Suppl. 1) 261-269

Liu ZB, Jiang JB, Yang HH, Jiang XM, Li JF (2019) Research Advance of Plant Heterosis. Mol Plant Breed 17: 4127-4134

Mohan Rao A (2001) Heterosis as a function of genetic divergence in sunflower (Helianthus annus L.). Ph D thesis submitted to Acharya N.G. Ranga Agricultural University, Hyderabad, 208pp

Mondal C, Sarkar S, Hazra, P (2019) Line × Tester analysis of combining ability in tomato (Lycopersicon esculentum Mill.). J Crop Weed 5(1):53-57.

Osei, Michael Kwabena, Danquah, Eric, Danquah, Agyemang, Blay, Esi, AduDapaah, Hans (2020) Hybridity testing of tomato F1 progenies derived from parents with varying fruit quality and shelf life using single nucleotide polymorphism (SNPs). Sci African (8)1-16

Panse VG, Sukathme PV (1967) Statistical methods for agricultural workers, ICAR, New Delhi, 145pp.

Pavan MP, Gangaprasad S (2022) Studies on mode of gene action for fruit quality characteristics governing shelf life in tomato (Solanum lycopersicum L.). Scientia Horticulturae 293. https://doi.org/10.1016/j.scienta.2021.110687

Roopa L, Sadhashiva AT, Reddy KM, Rao KPG, Prasad BCN (2001) Combining ability studies for long shelf life in tomato. Veg Sci 28:24-26

Shata Rupa Sinha, Ashutus Singha, Muhiuddin Faruquee, Md. Abu Sayem Jiku, Md. Arifur Rahaman, Md. Ashraful Alam and Mohammad Abdul Kader (2019) Post-harvest assessment of fruit quality and shelf life of two elite tomato varieties cultivated in Bangladesh. Bulletin of the National Research Centre 43:185

Singh AK, Asati BS (2011) Combining ability and heterosis studies in tomato under bacterial wilt condition. Bangladesh J Agric Res 36: 313-318

Singh RK, Chaudhary BD (1977) Biometrical Methods in Quantitative Genetic Analysis. Kalyani, Ludhiana

Somraj B, Reddy RVSK, Ravinder Reddy K, Saidaiah P, Thirupathi Reddy M (2018) Generation mean analysis of yield components and yield in tomato (Solanum lycopersicum L.) under high temperature conditions. J Pharmacogn Phytochem 7:1704-1708

Suo LJ, Lin SH, Qiang SZ (2010) Analysis on the major gene and polygene mixed inheritance of lycopene content in fresh consumptive tomato fruit. Hreditas 28 (4):458-462

Technow F (2019) Use of F2 bulks in training sets for genomic prediction of combining ability and hybrid performance. G3-Genes Genomes Genet 9:15571569

Page $18 / 19$ 
Turner JHJR (1953) A study of heterosis in upland cotton-I, yield of hybrids compared with varieties. Agron J 45: 487-490

Yadav SK, Singh BK, Baranwal DK, Solankey SS (2013) Genetic study of heterosis for yield and quality components in tomato (Solanum

lycopersicum). African J Agric Res 8(44): 5585-5591

Yogendra KN, Gowda PR (2013) Phenotypic and molecular characterization of a tomato (Solanum lycopersicum L.) F2 population segregation for improving shelf life. Genet Mol Res 12 (1):506-518

Yu KC, Wang H, Liu XG, Xu C, Li ZW, Xu XJ, Liu JC, Wang ZH, Xu YB (2020) Large-Scale Analysis of Combining Ability and Heterosis for Development of Hybrid Maize Breeding Strategies Using Diverse Germplasm Resources. Front Plant Sci 11:660 